\title{
A BTB-DOMAIN TRANSCRIPTION FACTOR RECRUITS CHROMATIN REMODELERS AND A HISTONE CHAPERONE DURING THE EXIT FROM PLURIPOTENCY
}

Daniel Olivieri ${ }^{1,}{ }^{*}$, Sujani Paramanathan ${ }^{1}$, Anaïs F. Bardet ${ }^{2}$, Daniel Hess ${ }^{1}$, Sébastien A. 5 Smallwood ${ }^{1}$, Ulrich Elling ${ }^{3}$, Joerg Betschinger ${ }^{1, *}$

$6{ }^{1}$ Friedrich Miescher Institute for Biomedical Research, Maulbeerstrasse 66, 4058 Basel,

7 Switzerland

$8{ }^{2}$ CNRS, University of Strasbourg, UMR7242 Biotechnology and Cell Signaling, Illkirch 67412, 9 France

$10{ }^{3}$ Institute of Molecular Biotechnology of the Austrian Academy of Science (IMBA), Vienna 11 BioCenter (VBC), Vienna, Austria

$12{ }^{*}$ Correspondence to: daniel.olivieri@fmi.ch, joerg.betschinger@fmi.ch

Transcription factors (TFs) harboring a btb (Broad-Complex, Tramtrack and Bric a brac) domain play important roles in development and disease. They are thought to recruit transcriptional modulators to DNA through their btb domain. However, a systematic molecular understanding of this TF family is lacking. Here, we identify the zinc finger btb-TF $Z b t b 2$ in a genetic screen for regulators of exit from pluripotency and dissect its mechanistic mode of action. We show that ZBTB2 binds the chromatin remodeler Ep400 to mediate downstream transcription. Independently, the btb domain directly interacts with the chromatin remodeller NuRD and the histone chaperone HiRA via the GATAD2A/B and UBN2 subunits, respectively. NuRD recruitment is a common feature of btb-TFs and we propose by phylogenetic analysis that this is an evolutionary ancient property. Binding to UBN2, in contrast, is specific to ZBTB2 and requires a C-terminal extension of the btb domain. This study therefore identifies a btb-domain TF that recruits chromatin modifiers and a histone chaperone during a developmental cell state transition, and defines unique and shared molecular functions of the btb-domain TF family. 


\section{INTRODUCTION}

2

Transcription factors (TFs) are key determinants of gene expression and, therefore, play a major role in development and disease (Lambert et al., 2018). TFs interpret the regulatory code of the genome by binding to DNA and regulating transcription (Lambert et al., 2018). While DNA-binding is well characterized (Weirauch et al., 2014), the mechanisms by which TFs modulate transcription are not completely understood. Many TFs present a modular protein architecture containing DNA-binding domains and domains that interact with transcriptional activators or repressors (Lambert et al., 2018). Zinc-finger domains (Znfs) are the most common family of DNA-binding domains and are often found in combination with Krueppel associated box (KRAB) or Broad-Complex, Tramtrack and Bric a brac (btb) domains (Collins et al., 2001). KRAB domains recruit KAP1 (Helleboid et al., 2019) and therefore mediate transcriptional repression. In contrast, there is no comprehensive understanding of the transcriptional role of btb domains.

There are three groups of TFs containing btb domains (btb-TFs): the Zbtb, the Bach, and the Nacc families, which are defined by their DNA binding domains: Znf, bZIP, and BEN, respectively (Stogios et al., 2005). In human and mouse, the Bach and the Nacc families contain only 2 members each, and the Zbtb family comprises 49 members. Several of them are critical regulators of fate allocation and differentiation across many organs and systems (Chevrier and Corcoran, 2014). A striking example is hematopoiesis, in which btb-TFs direct the differentiation of several lineages (Maeda, 2016). The btb domains are invariably found at the $\mathrm{N}$-terminus of btb-TFs and the DNA-binding domains at the C-terminus, separated by a long non-conserved linker region (Maeda, 2016). Although btb domains function in homoand heterodimerization and protein-protein interactions, the btb domains found in TFs and CUL3 ubiquitin ligases constitute separate families (Stogios et al., 2005). Mechanistic studies of Zbtb factors have focused on the proto-oncogenes Bcl6 (Zbtb27) and Lrf (Zbtb7a) and on the tumor suppressor Plzf (Zbtb16), and found that they act as transcriptional repressors via the recruitment of complexes such as NCOR/SMRT, BCOR, SIN3A/B, and NURD (Maeda, 2016).

Mouse embryonic stem cells (mESCs) are a developmentally relevant cell type that captures the pluripotent state of the pre-implantation mouse epiblast, and that recapitulates developmental progression upon release into differentiation in vitro (Martello and Smith, 2014). Furthermore, a large number of genomic datasets have been derived from mESCs, making them an ideal model for mechanistic studies of transcription. mESCs are maintained by provision of the cytokine leukemia inhibitory factor (LIF) in a fetal calf serum containing medium (Serum-LIF) (Smith et al., 1988) or of inhibitors (i) of glycogen synthetase kinase 3 (GSK3) and mitogen-activated protein kinase kinase (MEK) in a chemically defined medium (N2B27) (Ying et al., 2008). LIF, GSK3(i) and MEK(i) stabilize a pluripotency TF network centered on OCT4, SOX2 and NANOG (Martello and Smith, 2014). mESCs grown in the presence of the two inhibitors (2i) are called naïve and display higher and more homogeneous expression levels of pluripotency TFs than Serum-LIF grown cells (Silva and Smith, 2008). Naïve and Serum-LIF cell states are interconvertible (Galonska et al., 2015), while naïve cells can efficiently differentiate into post-implantation epiblast-like cells (EpiLCs) (Hayashi et al., 2011). In both naïve and Serum-LIF conditions, mESCs transition through a 2-cell-embryo (2C)like state that is thought to reflect properties of cleavage-stage embryos, and that is characterized by expanded developmental potency (Macfarlan et al., 2012), increased histone mobility (Bošković et al., 2014) and a specific gene expression signature which includes the 
upregulation of endogenous retroviruses (Macfarlan et al., 2011). While the TF Dux directly binds and activates the promoters of 2C-like genes (Hendrickson et al., 2017), it is less clear how other chromatin regulators, such as PRC1.6, Ep400 (Rodriguez-Terrones et al., 2018), or CAF-1 (Ishiuchi et al., 2015) mechanistically regulate 2C-like genes.

The Nucleosome Remodeling and Deacetylase (NuRD) complex is composed of HDAC1/2, CHD4, GATAD2A/B, RBBP4/7, MTA1/2/3, and MBD2/3 (Xue et al., 1998). MBD2 and MBD3 are mutually exclusive subunits and define the two functionally distinct MBD2-NuRD and MBD3-NuRD complexes (Guezennec et al., 2006). Mbd3 has been subject to several genetic studies in mESCs, showing that NuRD drives mESC differentiation (Kaji et al., 2007, 2006; Reynolds et al., 2012) and fine-tunes gene expression by modulating chromatin accessibility (Bornelöv et al., 2018).

The Histone Regulator A (HiRA) complex acts as the H3.3 histone chaperone at euchromatic loci (Goldberg et al., 2010). HiRA has been proposed to be recruited by naked DNA and to have a nucleosome-gap filling function (Ray-Gallet et al., 2011). It is composed of the subunits HIRA, CABIN1, and UBN1/2 (Tagami et al., 2004). It has been suggested that UBN1 and UBN2 are part of two independent, but functionally indistinguishable, complexes, UBN1-HiRA and UBN2-HiRA (Xiong et al., 2018). Euchromatic H3.3 is found in H2A.Z containing nucleosomes (Jin and Felsenfeld, 2007) that are incorporated into chromatin by the chromatin remodeling complex Ep400 (Pradhan et al., 2016), yet if and how Ep400 interacts with H3.3 chaperones is unclear. HiRA is required for exit from the naïve pluripotency during differentiation (Leeb et al., 2014), while the Ep400 complex is essential for the maintenance of mESCs (Fazzio et al., 2008).

Here, we identify the btb-TF Zbtb2 in a genetic screen for regulators of exit from mESC pluripotency, and report a detailed mechanistic analysis of its function, showing that ZBTB2 recruits ZNF639, MBD3-NuRD, UBN2-HiRA, and the Ep400 complex. Transcriptome analysis reveals that ZBTB2 interactors form two functionally distinct modules, one encompassing ZNF639, NuRD and HiRA, and the other corresponding to the Ep400 complex. We show that NuRD and HiRA associate with ZBTB2 via the subunits GATAD2A/B and UBN2, respectively. We systematically test these interactions across all btb-TFs in yeast-2-hybrid ( $Y 2 H$ ) screens. We find that ZBTB2 harbors an extension of the btb domain that mediates a unique interaction with UBN2. GATAD2A/B interaction is instead a common feature of btb-TFs and shared across several btb-TF phylogenetic branches, making NuRD recruitment a candidate ancestral feature of TF-associated btb domains. Our study therefore reveals unique and shared molecular functions of the btb-domain TF family.

\section{RESULTS}

\section{A sensitized genetic screen identifies Zbtb2 as regulator of the exit from pluripotency}

We performed a sensitized genetic screen for maintenance of pluripotency (Ying et al., 2003) in the presence of the GSK3 inhibitor CHIR99021 (CHIR) (Sato et al., 2004), which is unable to block mESC differentiation in the absence of LIF or Mek(i) (Wray et al., 2010). As the role of CHIR in mESCs maintenance is well characterized (Martello et al., 2012; Wray et al., 2011), this medium formulation should increase sensitivity for other, less understood, pathways. We mutagenized haploid mESCs (Elling et al., 2011) harboring an Oct4>GFP-puro reporter with 
retroviruses carrying a splicing acceptor site for insertional mutagenesis and Oct4 enhancer elements for overexpression (Schnütgen et al., 2008), leading to both loss and gain of function alleles. We cultured the mutagenized library in N2B27 +CHIR +puromycin to select for undifferentiated cells and harvested the cells after 16 to 23 days. We mapped the insertions by high-throughput inverse PCR and determined insertion enrichment compared to the starting library for every gene (Fig. 1A, Table S1). Confirming the specificity of our setup, Fgfr1 and Lif were among the highest scoring hits. FGFR1 is the main FGF receptor in mESCs and acts upstream of MEK activation (Molotkov et al., 2017), and chemical inhibition of FGFR signaling has been shown to substitute for MEK(i) in mESC maintenance (Ying et al., 2008). Lif, evidently a gain of function hit, is also able to sustain pluripotency in combination with CHIR (Sato et al., 2004). Oct4 is a technical false positive hit, as it can drive the expression of the Oct4>GFP-puro reporter irrespectively of the cell state. Confidence in our analysis was also bolstered by the identification of Esrrb and Tfcp2/1 amongst the insertion-depleted genes, as these are transcriptional mediators of CHIR activity (Martello et al., 2013, 2012; Qiu et al., 2015). Some of the highest scoring screen hits, such as Cbx1 (Mattout et al., 2015), Eed (Leeb et al., 2010; Tee et al., 2014), Trp53 (Lin et al., 2005) and Upf2 (Li et al., 2015), have previously been implicated in the exit from pluripotency. We therefore decided to validate the hits Zbtb2, Zfp42, Nexmif, and Nmt1.

The Nexmif gene lies upstream of the Rlim transcription start site and RLIM is a known E3 ubiquitin ligase for ZFP42 (Gontan et al., 2012), so we reasoned that the Nexmif insertion enrichment would lead to Rlim overexpression. We therefore generated CRISPR knock-out clones (Table S2) for Zfp42 (Fig. S1A), Nmt1 (Fig. S1B) and Zbtb2 (Fig. S1C), and Rlim overexpressing cells using naïve TNG-A mESCs, a conventional diploid cell line harboring a Nanog $>$ GFP reporter (Chambers et al., 2007). Upon exposure to N2B27 +CHIR, concomitant with the addition of recombinant basic FGF (bFGF) to increase the stringency of the assay, all three mutants and the Rlim overexpressing cells showed delayed down-regulation of the Nanog reporter when compared to wildtype (WT) cells (Fig. 1B, C, D, and Fig. S1D), indicating that they are bona fide loss of function screen hits. Overexpression of Rlim in the Zfp42 mutant did not modify the phenotype of the $Z f p 42$ single mutant (Fig. 1B and Fig. S1D), supporting the epistatic relationship between Rlim and Zfp42. While Zfp42 overexpression did not affect differentiation (Fig. 1B and Fig. S1D), strong Zbtb2 constitutive overexpression using a CAG promoter caused cell death (not shown). We therefore tested the effect of moderate $Z b t b 2$ overexpression, achieved by a doxycycline (DOX) inducible promoter, and observed accelerated differentiation upon Zbtb2 induction (Fig. 1D and Fig. S1D).

Nmt1 encodes an N-myristoyltransferase (Yang et al., 2005) and myristoylation is required for the function of FRS2, an essential component for FGFR1 signaling (Kouhara et al., 1997). We therefore speculated that loss of Nmt1 would inhibit mESC differentiation by dampening mitogen activated protein kinase (MAPK) signaling. However, ERK phosphorylation upon exposure to bFGF was unperturbed in $\mathrm{Nmt1}^{-/}$cells (Fig. S1E). We therefore turned our attention to another myristoylated protein, LAMTOR1 (Thinon et al., 2014), which is required for TFE3 nuclear exclusion (Villegas et al., 2019) and, in turn, for the exit from pluripotency (Betschinger et al., 2013). Immunofluorescence staining revealed abnormal nuclear localization of TFE3 in $\mathrm{Nmt1}^{-/} \mathrm{mESCS}$ (Fig. 1E), suggesting that ectopically active TFE3 mediates the differentiation delay in the absence of Nmt1.

Although Zbtb2 had already been suggested to play a role in the differentiation of Serum-LIF mESCs (Karemaker and Vermeulen, 2018), we decided to focus our efforts on this factor in 
the hope of gaining insights that are broadly applicable to btb-TFs. We first sought to better characterize $Z b t b 2$ 's role in the exit from the naïve state and determine when the earliest developmental defect would arise. We first tested differentiation of 2iLIF cells using the EpiLC differentiation protocol, which faithfully mimics the pre- to post-implantation epiblast transition (Hayashi et al., 2011), and observed a delay in Nanog reporter downregulation in Zbtb2 ${ }^{--}$cells although Nanog levels were unchanged in steady-state 2iLIF cultures (Fig. $1 \mathrm{~F}$ and Fig.S1F). We then turned to differentiation in Serum-LIF, which establishes a developmentally advanced pluripotent state (Kalkan and Smith, 2014). Even in this assay, Nanog>GFP downregulation was delayed in $\mathrm{Zbtb2}^{-/}$cells (Fig. $1 \mathrm{~F}$ and Fig.S1F). To determine transcriptome-wide changes we performed RNA sequencing (RNAseq) of WT and Zbtb2 ${ }^{-/}$cells in 2iLIF and during differentiation. We found that gene expression changes that accompany the 2iLIF to Serum-LIF transition in WT cells were dampened in Zbtb2 $2^{-/}$cells exposed to Serum-LIF for 48 hours ( $h$ ) (Pearson correlation coefficient $R=-0.19$, Fig. 1G, Table S3). When we specifically focused on genes regulated during embryonic development (Boroviak et al., 2015), we found that $\mathrm{Zbtb2}^{-/}$cells in Serum-LIF, when compared to WT controls, were impaired in upregulating genes that are expressed in the post-implantation epiblast and in downregulating genes that are predominantly transcribed in the pre-implantation epiblast (Fig. 1G). This coherent deregulation of developmental genes was specific to the Serum-LIF transition and undetectable in steady-state 2iLIF cells (Fig. S1G). In summary, loss of Zbtb2 delays and $Z b t b 2$ overexpression increases $\mathrm{mESC}$ differentiation, demonstrating an instructive role of ZBTB2 in cell state transitions.

An extended btb domain binds UBN2 and GATAD2B; NuRD interaction is stabilized by ZNF639.

To understand the mechanisms by which $Z$ btb2 exerts its function, we performed affinity purification-mass spectrometry (AP-MS) of ZBTB2 in mESCs. In the absence of antibodies detecting the endogenous protein we generated mESCs expressing an avidin (AVI)-tagged Zbtb2 transgene which, similar to untagged Zbtb2, induces differentiation when overexpressed (Fig. S2A), therefore confirming functionality. Using streptavidin pull-downs, we identified ZBTB25 and ZNF639, previously reported to interact with ZBTB2 (Karemaker and Vermeulen, 2018), and all subunits of the NuRD and of the HiRA complexes as specific ZBTB2 interactors (Fig. 2A). We did not detect MBD2 or UBN1 peptides, demonstrating copurification of MBD3-NuRD and UBN2-HiRA complexes, specifically. To better understand how ZBTB2 recruits such a complex interactome, we performed AP-MS of ZBTB2-AVI alleles harboring mutations in the btb and Znf domains (Table S4). All experiments were carried out in a $\mathrm{Zbtb2}^{-/} \mathrm{Zbtb25^{-- }}$ background (Fig. S2B) to prevent indirect interactions due to bait dimerization with endogenous ZBTB2 or ZBTB25, and in the presence of Benzonase nuclease to avoid DNA- and RNA-bridged interactions. Mutation of the btb domain caused loss of the interaction with UBN2-HiRA and of Znf1 abolished the interaction with ZNF639 and NuRD, while mutation of the other Znfs did not significantly alter the interactome (Fig. 2B, Table S5). However, loss of interaction upon domain mutation does not imply direct physical binding, as it could be due to an indirect functional dependency or bridging factor.

We, therefore, turned to yeast-two-hybrid $(\mathrm{Y} 2 \mathrm{H})$ assays. First, we addressed dimerizing properties of ZBTB2. We detected homodimerization of ZBTB2's btb domain and heterodimerization with ZBTB25 (Fig. 2C, D). To our surprise, we also found a strong interaction of ZBTB2's linker region with ZBTB2's btb domain but not with the full-length ZBTB2 protein (Fig. 2C, D). The linker is 136 amino acid in length and predicted to be 
unstructured. We generated linker region deletions and found that the segment immediately adjacent to the btb domain (link-D1) mediates interaction with the btb domain (Fig.2C, E), suggesting the existence of an extended btb domain structure. We therefore used this extended btb domain (btb-link) in further assays. Next, we tested the direct interaction of ZBTB2 with the HiRA complex subunits HIRA, CABIN1, and UBN2. We found that UBN2, but not HIRA or CABIN1, binds full-length ZBTB2 and the btb-link domain, but neither the isolated btb domain or linker region (Fig. S2C). Deletion analysis of UBN2 identified a minimal interacting region of 68 amino acids that is outside of annotated domains (Fig. S2D, E). The btb-link domain therefore mediates homo- and heterodimerization with ZBTB2 and ZBTB25, respectively, and interaction with UBN2.

We then tested binding to the NuRD subunits RBBP4, MBD3, MTA2, MTA3, HDAC1, GATAD2A, and GATAD2B. Based on our AP-MS results (Fig. 2B), we expected that Znf1 would mediate such an interaction, yet the only direct interactions we identified were between GATAD2A and ZBTB2, and between GATAD2B and both, ZBTB2 and the btb-link domain (Fig. S2F, G). No interactions were found with $\mathrm{Znf1}$, the btb domain or the linker region. Additional $\mathrm{Y} 2 \mathrm{H}$ assays revealed that the C-terminal half of GATAD2B, which includes its Gata-type Znfs, mediates interaction with the btb-link domain (Fig. $\mathrm{S} 2 \mathrm{H}, \mathrm{I}$ ).

As the direct interactions with both UBN2 and GATAD2B were mediated by the btb-link domain, but not the btb domain or linker alone, we sought to determine the minimal btb domain extension required for either interaction. Through serial truncations we found that a 44 amino acid extension was necessary for both (Fig. 2C, F). This, together with the direct interaction between the conserved btb domain and this 44 amino acid fragment (Fig. 2C, E) strongly suggests a functionally essential structural extension of ZBTB2's btb domain.

Further $\mathrm{Y} 2 \mathrm{H}$ assays identified a direct interaction between ZNF639 and ZBTB2's Znf1 domain (Fig. 2G), as expected by the AP-MS data (Fig. 2B). Znf1 was required for the interaction with NuRD in AP-MS (Fig. 2B), but did not mediate any direct interaction with NuRD subunits (Fig. S2F, G). Therefore, we wondered whether ZNF639 is required to stabilize or enhance the interaction between NURD and ZBTB2, which is mediated by the extended btb domain. To test this hypothesis, we performed AP-MS of ZBTB2-AVI in wt and Znf639- mESCs (Fig. S2J, Table S2), and found a $\sim 8$-fold reduction in NuRD interaction upon loss of Znf639 (Fig. 2H, I).

In summary, our AP-MS and $\mathrm{Y} 2 \mathrm{H}$ experiments show that the btb domain of ZBTB2 mediates homodimerization and heterodimerization with ZBTB25, that an extended btb domain recruits the UBN2-HiRA complex through the UBN2 subunit, and that the interaction with MBD3-NuRD is direct via btb-link binding GATAD2A/B, but also requires Znf1 recruiting ZNF639.

Recruitment of HiRA is a unique property of ZBTB2, while GATAD2A/B interaction is a conserved feature of TF-associated btb domains.

Interaction of a btb-TF with the HiRA complex has not been reported before, while the interaction with NuRD has been previously shown for ZBTB7A (Masuda et al., 2016). We therefore wondered if HiRA and NuRD recruitment would be conserved across btb-TFs. We first attempted to identify additional HiRA-interacting btb domain proteins by performing UBN2 and UBN1 AP-MS in mESCs. UBN2 pull-down identified the HiRA components CABIN1, HIRA, and UBN1. The only TFs we recovered were ZBTB2 and its partner ZNF639 (Fig. 3A), but 
not ZBTB25. In the UBN1 AP-MS we detected ZBTB2 and MEF2D (Fig. S3A), a known direct interactor of CABIN1 (Youn et al., 1999).

As not all btb-TFs are expressed in mESCs, we turned to $\mathrm{Y} 2 \mathrm{H}$ to systematically assay ability to bind to UBN2 or GATAD2A/B. We performed Y2H screens of the btb domains of the $49 \mathrm{Zbtb}$ factors, Nacc1/2, and Bach1/2, extending the conserved btb domains by at least 60 amino acids, in case other btb domains would possess extended structures similar to Zbtb2. Strikingly, only ZBTB2's btb-link domain interacted with UBN2 (Fig. 3B, Fig. S3B), corroborating the UBN1/2 AP-MS data to support that HiRA recruitment by ZBTB2 is unique. Surprisingly, we identified btb domains of 14 btb-TFs to interact with GATAD2A or GATAD2B (Fig. 3C, D, Fig. S3B, C). We were not able to confirm binding to ZBTB7A's btb domain (Masuda et al., 2016) due to autoactivation in the $\mathrm{Y} 2 \mathrm{H}$ assays (Fig. S3B, C). Taken together, at least 15 out of 54 btb-TFs bind to NuRD subunits, suggesting that GATAD2A/B interaction is a common function of TF-associated btb domains.

We wondered whether the GATAD2A/B interacting domains would be phylogenetically related. Phylogenies based on the complete sequence of Zbtb TFs do not reflect the similarities within the btb domains (Siggs and Beutler, 2012) because of the influence of the Znf domains. We therefore constructed a phylogenetic tree based on btb domain sequences (Fig. 3E, Table S6). Although confidence for the evolutionary older branches was low, GATAD2A/B -interacting btb domains do not form a clade, but are scattered throughout the tree, including the Bach and Nacc clades. This shows that GATAD2A/B recruitment is an ancestral property of TF-associated btb domains.

To test for heterodimerization with ZBTB2 and if this correlates with binding to GATAD2A/B, we repeated the $\mathrm{Y} 2 \mathrm{H}$ btb domain family screen using ZBTB2's btb domain as bait (Fig. 3F, Fig. S3B). This identified 6 btb domains heterodimerizing with ZBTB2, of which 3 also bound to GATAD2A/B. Specificity for heterodimerization and GATAD2A/B binding are therefore separate properties of btb domain.

Although ZBTB25's btb domain heterodimerizes with ZBTB2, it did not bind UBN2 or GATAD2A/B (Fig. 3B-F). We therefore hypothesized that binding to ZBTB25 is not relevant to ZBTB2's role in cell fate transition. To test this, we characterized the phenotype of $Z$ btb25-and $\mathrm{Zbtb2} 2^{--} \mathrm{Zbtb25} \%$ mESCs (Fig. S3D) in the 2iLIF to Serum-LIF transition and compared it to $Z b t b 2^{--}$cells. In fact, loss of Zbtb25 did not delay Nanog>GFP downregulation or modify the phenotype of $Z b t b 2$ single mutants (Fig. S3E, F).

In summary, we tested the conservation of UBN2 and GATAD2A/B interaction by AP-MS and $\mathrm{Y} 2 \mathrm{H}$. While UBN2 recruitment appears to be unique to ZBTB2, GATAD2A/B interaction is a common and ancestral feature of btb-TFs.

\section{ZBTB2 interacts with the Ep400 complex in a HiRA-independent manner}

While evaluating the functionality of tagged Zbtb2 constructs for AP-MS, we found that overexpression of ZBTB2 fused with an extended C-terminal 3xHA-AVI-3xFLAG-tag (HAF-tag) caused a delay in Nanog $>$ GFP reporter downregulation (Fig. 4A). This phenotype is opposite to ZBTB2-AVI overexpression but similar to loss of Zbtb2, suggesting that ZBTB2-HAF acts dominant negative. To understand the underlying molecular defect, we compared the interactomes of the ZBTB2-AVI and ZBTB2-HAF fusion proteins. To our surprise, we found that the entire Ep400 complex copurified with the dominant negative ZBTB2-HAF, while none of 
the other interactors was lost (Fig. 4B). This prompted us to look more carefully at the ZBTB2AVI AP-MS data and consistently found Ep400 subunit peptides across independent experiments (Table S5), suggesting that ZBTB2-HAF stabilizes a physiological, but transient or weak interaction.

Ep400 incorporates H2A.Z/H3.3 histones into chromatin (Pradhan et al., 2016). We therefore hypothesized that association of Ep400 with ZBTB2 could be mediated by the HiRA complex, which is an H3.3 chaperone (Tagami et al., 2004). To test this, we performed AP-MS of ZBTB2HAF in wt and in Ubn2 ${ }^{-/-}$mESCs (Fig. S4A). As expected, lack of UBN2 caused loss of the HiRA interaction, but the association with Ep400 was not affected (Fig. S4B). Interestingly, we noted a substantial increase of ZBTB25 in ZBTB2 AP-MS in Ubn2\% cells (Fig. S4B). UBN2 and ZBTB25 may therefore compete for interaction with ZBTB2. Together with the inability of ZBTB25 to interact with UBN2 (Fig. 3B), this supports the idea that ZBTB25 is a negative regulator of HiRA recruitment by ZBTB2. We therefore conclude that ZBTB2 interacts weakly or transiently with Ep400 and independent of HiRA co-binding.

\section{Ep400 and Znf639/NuRD/HiRA constitute independent Zbtb2 functional modules}

To address the functional role of ZBTB2's protein interactions, we set out to compare the loss of function phenotype of Zbtb2 with that of its binding partners. Depletion of the Ep400 complex subunits Ep400 and Kat5 causes loss of mESC self-renewal (Fazzio et al., 2008), while knockout of Mbd3 (Kaji et al., 2006) or Hira (Leeb et al., 2014) induces resistance to exit from the $\mathrm{mESC}$ state during differentiation. However, these phenotypes may arise from pleiotropic, Zbtb2-unrelated roles. As the ZBTB2-HiRA interaction is dependent on UBN2, and the ZBTB2NuRD interaction is stabilized by ZNF639, we reasoned that analysis of $\mathrm{Ubn} 2^{-/-}$and $\mathrm{Znf639^{-/ }}$ cells might reveal Zbtb2-specific functions. Compared to Zbtb2 mutants, Znf639-/- and Ubn2 ${ }^{-/-}$ mESCs showed moderate delays in Nanog>GFP downregulation upon Serum-LIF transition (Fig. 5A, B). Although weak, these phenotypes are consistent with Znf639 and Ubn2, and by extension NuRD and HiRA, cooperating with Zbtb2 in cell fate transitions.

To investigate these functional relationships further, we performed RNAseq of $\mathrm{Zbtb2} \%$, Znf639 $\%$ and $\mathrm{Ubn2}^{-}$mESCs after $48 \mathrm{~h}$ in Serum-LIF. Consistent with Nanog reporter phenotypes, pre-implantation epiblast-enriched transcripts were upregulated and postimplantation epiblast-specific genes were downregulated in Znf639 and Ubn2 mutant cells in Serum-LIF, although to a lesser extent than observed in Zbtb2 knockout cells (Fig. S5A). To describe Zbtb2's transcriptional role, we focused on genes significantly changing upon loss of Zbtb2 and included published transcriptome data of $\mathrm{Mbd3}^{-/}$(Reynolds et al., 2012), Ep400 and Kat5 knock-down (KD) (Acharya et al., 2017; Fazzio et al., 2008) mESCs in Serum-LIF (Table S3). We found that transcriptional alterations in $\mathrm{Zbtb2}^{-/}$cells correlated with those upon knockdown of $E p 400(\mathrm{R}=0.39)$ and $K a t 5(\mathrm{R}=0.37$ and 0.36$)$, showing a mechanistic relationship between Zbtb2 and the Ep400 complex (Fig. 5C). Changes in Mbd3 mutants, in contrast, correlated with those in Znf639 knockout cells ( $R=0.36)$, corroborating the strong reduction of NuRD binding to ZBTB2 in Znf639 mutants. Furthermore, alterations in Mbd3, Znf639, and Ubn2 mutants correlated reciprocally, pointing towards a functional ZNF639/NuRD/HiRA unit (Fig. 5C). Strikingly, there was no general correlation between Zbtb2/Ep400-dependent and Znf639/Mbd3/Ubn2-dependent gene expression, raising the hypothesis that Ep400 and ZNF639/NuRD/HiRA cooperate with ZBTB2 within functionally independent modules. 
1 For more detailed insight, we performed k-means clustering of differential gene expression

2 (Fig. 5D). This identified cluster 1 genes to be upregulated in Zbtb2 $2^{-/}$and Kat5 KD cells and 3 downregulated in $\mathrm{Znf639} \%, \mathrm{Mbd3}^{-\%}$, and $\mathrm{Ubn} 2^{-\%}$ cells. Cluster 1 contains the strongest 4 changing genes in $\mathrm{Zbtb}^{-/}$cells and closer inspection revealed them to be enriched for $2 \mathrm{C}$-like 5 genes, as corroborated by the correlation with DUX-induced genes (Fig. 5D) (Hendrickson et 6 al., 2017). Such opposite functions are consistent with independent complexes competing for 7 limiting ZBTB2 amounts, where loss of the ZNF639/NuRD/HiRA complex would lead to an 8 increase in Ep400/ZBTB2 dependent 2C-like gene repression.

9 Taken together, our findings show that Zbtb2, Znf639 and Ubn2 loss of function cause a 10 developmental delay that is reflected in the deregulation of pre- and post-implantation epiblast specific genes. However, a systematic analysis of ZBTB2 target genes revealed that gene expression changes upon loss of Ep400 and Kat5 correlate best with Zbtb2 mutants, while alterations upon loss of Znf639, Ubn2, and Mbd3 correlate reciprocally, but not with Zbtb2 mutants. This suggests the existence of two functionally distinguishable ZBTB2 modules, one associated with the Ep400 complex, causing most Zbtb2-dependent gene expression changes, and the other with ZNF639/NuRD/HiRA. Opposite roles in regulating 2Clike genes expression suggest that these modules act antagonistically within one protein complex, or independently in competing biochemical complexes.

ZBTB2 inhibits 2C-like gene transcription indirectly but binds and represses its own promoter

The Ep400 complex represses expression of 2C-like genes without binding to their regulatory DNA sequences (Rodriguez-Terrones et al., 2018). We therefore wondered if ZBTB2 is similarly depleted at 2C-like genes. As ZBTB2 mostly binds to promoters (Karemaker and Vermeulen, 2018), we analyzed ZBTB2, EP400, KAT5 (Chen et al., 2015), MBD3, and CHD4 (Bornelöv et al., 2018) occupancy at promoters of genes belonging to cluster 1 (2C-like genes) or clusters 2-9 (Fig. 5E, F, and Fig. S5B, C). We found that these factors are depleted at cluster 1 promoters, as opposed to DUX which is highly enriched, as expected (Hendrickson et al., 2017). Therefore, 2C-like gene repression by ZBTB2/Ep400 is either indirect through the regulation of other genes, or mediated through DNA-binding independent mechanisms.

Nevertheless, ZBTB2 is enriched at promoters, where it colocalizes with EP400, KAT5, MBD3, and CHD4 (Fig. S5D). Therefore, we wondered what the activity of ZBTB2 on bound genes is. One of the most prominent ZBTB2 ChIP-seq peaks lays on the promoter of Zbtb2 itself (Fig. $\mathrm{S} 5 \mathrm{E})$. As some of our Zbtb2 mutants generated Zbtb2 transcripts that were detectable by quantitative polymerase chain reaction (qPCR) (Fig. S1C, Fig. S5E), this gave us the opportunity to test the activity of ZBTB2 on its own promoter. Mutation of Zbtb2 leads to increased Zbtb2 transcript levels and overexpression of a Zbtb2 construct not detected by our qPCR probes leads to decreased endogenous Zbtb2 transcript levels (Fig. S5F). In conclusion, ZBTB2 represses transcription either via promoter binding, as is the case for autoregulation, or via indirect mechanisms, as is the case for $2 \mathrm{C}$-like genes. 
In this study, we exploited a genetic screen in mESCs to mechanistically and functionally characterize the btb-TF ZBTB2, gaining insights that are broadly applicable to btb-TFs.

We made use of a sensitized set-up and took advantage of a loss-of-function and gain-offunction haploid mESC library, identifying previously described and novel regulators of exit from pluripotency (Fig. 1A). We validated the role of Zfp42/Rlim, Nmt1, and Zbtb2 in an independent cell line and using an unrelated reporter (Fig. 1B, C, D), confirming their role in mESC differentiation. Previous studies of Zfp42 (Masui et al., 2008; Scotland et al., 2009) led to the prevailing idea that $Z f p 42$ is dispensable for pluripotency or development, to the point that $Z f p 42^{-/}$cells have been used in genetic screens for the exit from pluripotency (Leeb et al., 2014; Villegas et al., 2019; Yang et al., 2012). Our data also supports the relationship between Rlim and Zfp42 (Gontan et al., 2012) and calls for further investigation of the function of this genetic axis in pluripotency. Nmt1 is the major N-Myristoyltransferase in mESCs and is essential in early mouse development (Yang et al., 2005). As some of its substrates are known (Thinon et al., 2014), we tested two possible modes of action, via FRS2 (Kouhara et al., 1997) and MAPK-signaling, or via LAMTOR1 and TFE3 localization (Villegas et al., 2019). While we were not able to detect changes in ERK phosphorylation (Fig. S1E), we noticed a clear increase in nuclear TFE3 (Fig. 1E), which is compatible with a TFE3-dependent differentiation delay.

With the aim of gaining fundamental insights into transcriptional regulation of cell state transition, we focused our work on the btb-TF $Z b t b 2$. A previous report showed morphological delay upon LIF removal in $\mathrm{Zbtb2}^{-}$cells (Karemaker and Vermeulen, 2018), but did not characterize this phenotype further. We found that $\mathrm{Zbtb}^{-\%}$ naïve $\mathrm{mESCs}$ are defective in differentiating into the EpiLC and Serum-LIF cell states (Fig. 1F). Upon transitioning to Serum-

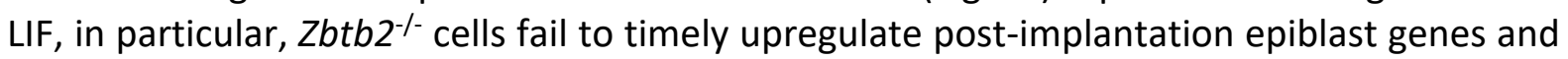
to down-regulate pre-implantation epiblast genes (Fig. 1G). To understand the underlying molecular mechanism, we performed a thorough biochemical analysis of ZBTB2-containing protein complexes and showed (1) that ZBTB2's btb domain mediates homodimerization, heterodimerizaton with ZBTB25, interaction with UBN2-HiRA via UBN2, and interaction with MBD3-NuRD via GATAD2A/B, (2) that ZBTB2 first Znf interacts with ZNF639 and that this interaction is required to establish or stabilize binding of ZBTB2 to NuRD, and (3) that ZBTB2 interacts weakly or transiently with the Ep400 complex. Ep400 incorporates H3.3 containing histones into chromatin (Pradhan et al., 2016), for which HiRA is a chaperone (Tagami et al., 2004). Nevertheless, we show that Ep400 recruitment to ZBTB2 is independent of HiRA (Fig. S4B). Although we identified binding to Ep400 using a dominant negative ZBTB2 construct (ZBTB2-HAF), we propose that this is a physiologically relevant interaction, because: (1) low levels of Ep400 subunits are detectable in AP-MS of the functional ZBTB2-AVI construct (Table S5), (2) transcriptional changes upon loss of Zbtb2 and Ep400/Kat5 correlate (Fig. 5C, D), (3) a direct interaction between ZBTB2 and the Ep400 subunit KAT5 was identified by highthroughput $\mathrm{Y} 2 \mathrm{H}$ screening (Luck et al., 2020).

These molecular insights have important implications for our understanding of histone variant deposition. The prevailing models for HiRA recruitment hypothesize a transcription-coupled mechanism (Sarai et al., 2013) or intrinsic affinity for nucleosome-free DNA (Ray-Gallet et al., 2011). As ZBTB2 is able to recruit HiRA and to localize at promoters, we propose that HiRA 
recruitment at transcription start sites can be mediated by ZBTB2. The HiRA complex binds H3.3 (Tagami et al., 2004), yet there is no known HiRA-associated chromatin remodeler for H3.3 deposition, such as ATRX for the H3.3 chaperone DAXX (Grover et al., 2018). Our work provides the first physical link between HiRA and a chromatin remodeler. The hypothesis that HiRA might be coupled to Ep400 is further supported by Ep400's preference for H2A.Z/H3.3 nucleosome deposition (Pradhan et al., 2016).

While btb domain-containing TFs are acknowledged to play biologically important and disease-relevant roles (Chevrier and Corcoran, 2014), a general understanding of their evolution and molecular function is lacking. Building on our characterization of ZBTB2's interactome, we systematically assayed the biochemical properties of btb-TFs. Using $\mathrm{Y} 2 \mathrm{H}$ we found that UBN2 recruitment is a unique feature of ZBTB2. GATAD2A/B binding, instead, is shared by at least 15 btb-TFs and is therefore the most common feature of btb-TFs reported to date. Instances of this interaction are present in the Nacc family, in the Bach family, and in many apparently unrelated branches of the Zbtb family (Fig. 3E), suggesting that GATAD2A/B binding is an ancestral property of btb-TFs. Intriguingly, the Human Reference Protein Interactome Mapping Project (Luck et al., 2020) identified the btb-TFs ZBTB1, ZBTB2, ZBTB8A, ZBTB14, and BACH2 to directly interact with KAT5, suggesting that Ep400 recruitment might also be a conserved property of btb-TFs. The dimerization specificity of btb domains is incompletely understood and has been proposed to be enforced by a quality control mechanism (Mena et al., 2018). In contrast, our Y2H data for ZBTB2 heterodimerization shows that dimerization specificity is a btb domain-intrinsic property and unrelated to interaction with other partners. For example, ZBTB25's btb domain heterodimerizes with ZBTB2's btb domain (Fig. 3F), but does not interact with GATAD2A/B or UBN2, while ZBTB2's btb domain does so (Fig. 3B-D). This raised the possibility that ZBTB25 might modulate ZBTB2's avidity for HiRA and NuRD, which was confirmed, in the case of HiRA, by the competition between UBN2 and ZBTB25 for ZBTB2 binding (Fig. S4B). Nevertheless, this regulatory mechanism does not seem to play an important role, as the lack of $Z b t b 25$ does not affect differentiation in either WT or Zbtb2 $2^{-/}$cells (Fig. S3E, F).

The linker region between the btb domain and the first Znf of ZBTB proteins is not conserved (Stogios et al., 2005) and, although it can mediate protein interactions, is usually considered to work as a flexible unstructured linker (Maeda, 2016). We found that the first 44 amino acids of ZBTB2's linker interact with the conserved btb domain, but not with the extended btb domain (Fig. 2E), and that extension by these 44 amino acids is necessary for the btb domain to bind UBN2 and GATAD2B (Fig. 2F). We interpret this as evidence for a structured extension of the btb domain of ZBTB2. As ZBTB7A does not require a btb domain extension to interact with GATAD2B (Masuda et al., 2016), we hypothesize that this is a unique feature of ZBTB2 that evolved with its ability to bind UBN2.

To assess the function of the characterized ZBTB2 interactions, we generated Znf639/- and $\mathrm{Ubn} 2^{-/-} \mathrm{mESCs}$ and analyzed their differentiation phenotypes and transcriptional alterations. Znf639 $\%$ and Ubn2 ${ }^{-/}$mESCS show a delay in Nanog $>$GFP downregulation (Fig. 5A, B) that, although weaker than in Zbtb2 mutants, is consistent with the reported phenotypes of $\mathrm{Mbd3}^{-}$ 1- (Kaji et al., 2006) and Hira ${ }^{-/}$cells (Leeb et al., 2014). At the transcriptional level, Mbd3, Ubn2 and Znf639 mutants correlate reciprocally (Fig. 5C), behaving as a functional unit, which is consistent with the role of ZNF639 in stabilizing NuRD interaction (Fig. $2 \mathrm{H}$ ). Although we do not know whether ZNF639, UBN2 and NuRD simultaneously bind ZBTB2, this suggests that they work synergistically, rather than regulating independent genes. Znf639/NuRD/HiRA 
1 module mutants show no transcriptional correlation with $\mathrm{Zbtb2}{ }^{--}$cells, suggesting that ZBTB2

2 directs gene regulation predominantly through another interactor, such as the Ep400

3 complex. In fact, Zbtb2-specific expression changes correlate with those upon Ep400 and Kat5

4 depletion (Fig. 5C), pointing to the existence of two separate ZBTB2 effector modules, one

5 associated with Ep400, and the other with ZNF639/NuRD/HiRA. The phenotypic convergence

6 of Zbtb2, Znf639, Mbd3 and Ubn2/Hira on promoting exit from naïve pluripotency might be

7 due to coherent regulation of pre- and post-implantation specific genes (Fig. S5A). The role of

8 Ep400 in driving mESC differentiation remains to be determined, since Ep400 and Kat5 are

9 essential for ESC self-renewal (Fazzio et al., 2008).

10 ZBTB2 and the Ep400 complex (Rodriguez-Terrones et al., 2018) repress 2C-like genes, while ZNF639, MBD3, and UBN2 promote their transcription (Fig. 5D). Nevertheless, all these factors are depleted at the promoters of $2 \mathrm{C}$-like genes (Fig. 5E, F, Fig. S5B, C), demonstrating that this regulation is indirect. The underlying mechanism remains to be determined. Since the H3.3 histone chaperone DAXX/ATRX directly inhibits 2C-like gene expression (Elsässer et al., 2015; Sadic et al., 2015), this mechanism may involve ZBTB2 modulating H3.3 dynamics through Ep400 and HiRA. A similar mechanism may contribute to the regulation of 2C-like genes by the canonical H3 chaperone CAF1 (Ishiuchi et al., 2015). However, ZBTB2 can also repress transcription in a sequence-specific and direct manner, which we demonstrate by taking advantage of a prominent ZBTB2 ChIP-seq peak at the Zbtb2 promoter and showing that ZBTB2 regulates its own transcription in a negative feedback-loop (Fig. S5F).

In summary, this study presents a detailed biochemical and transcriptional analysis of ZBTB2, and identifies how chromatin modifiers and histone chaperones are recruited by this TF during cell state transition. We use these molecular insights to systematically analyze btb-TFs and to propose a comprehensive concept for their evolution and function. This work will serve as a resource for the study of btb-TFs and inspire further systematic approaches to this important family of TFs. 


\section{ACKNOWLEDGMENTS}

2

3 We thank Hans-Rudolf Hotz (FMI) for help with the phylogenetic analysis; H. Kohler (FMI) for 4 cell sorting; V. lesmantavicius (FMI) for help with computational analysis of mass 5 spectrometry data; Min Jia and Jeff Chao (FMI) for providing LIF; Austin Smith (University of 6 Exeter) for providing TNG-A cells. D.O. was supported by EMBO (ALTF 1632-2014) and Marie 7 Curie Actions (LTFCOFUND2013, GA-2013-609409). Research in the lab of J.B. is supported by 8 the Novartis Research Foundation.

10 AUTHOR CONTRIBUTIONS

11

12 D.O. and J.B. conceived the study; D.O. designed, performed, and analyzed experiments; S.P. 13 performed $\mathrm{Y} 2 \mathrm{H}$ experiments; D.H. mass spectrometry; U.E. haploid mESCs generation and mutagenesis; A.F.B. analyzed the screen data; S.A.S. supervised sequencing library generation and sequencing; J.B. performed computational analysis; D.O. wrote the paper.

\section{COMPETING INTERESTS}

18

The authors declare no conflict of interest. 


\section{REFERENCES}

Acharya D, Hainer SJ, Yoon Y, Wang F, Bach I, Rivera-Pérez JA, Fazzio TG. 2017. KATIndependent Gene Regulation by Tip60 Promotes ESC Self-Renewal but Not Pluripotency. Cell Reports 19:671-679. doi:10.1016/j.celrep.2017.04.001

Betschinger J, Nichols J, Dietmann S, Corrin PD, Paddison PJ, Smith A. 2013. Exit from Pluripotency Is Gated by Intracellular Redistribution of the bHLH Transcription Factor Tfe3. Cell 153:335-347. doi:10.1016/j.cell.2013.03.012

Bornelöv S, Reynolds N, Xenophontos M, Gharbi S, Johnstone E, Floyd R, Ralser M, Signolet J, Loos R, Dietmann S, Bertone P, Hendrich B. 2018. The Nucleosome Remodeling and Deacetylation Complex Modulates Chromatin Structure at Sites of Active Transcription to Fine-Tune Gene Expression. Mol Cell 71:56-72.e4. doi:10.1016/j.molcel.2018.06.003

Boroviak T, Loos R, Lombard P, Okahara J, Behr R, Sasaki E, Nichols J, Smith A, Bertone P. 2015. Lineage-Specific Profiling Delineates the Emergence and Progression of Naive Pluripotency in Mammalian Embryogenesis. Dev Cell 35:366-382. doi:10.1016/j.devcel.2015.10.011

Bošković A, Eid A, Pontabry J, Ishiuchi T, Spiegelhalter C, Ram EVSR, Meshorer E, TorresPadilla M-E. 2014. Higher chromatin mobility supports totipotency and precedes pluripotency in vivo. Gene Dev 28:1042-1047. doi:10.1101/gad.238881.114

Chambers I, Silva J, Colby D, Nichols J, Nijmeijer B, Robertson M, Vrana J, Jones K, Grotewold L, Smith A. 2007. Nanog safeguards pluripotency and mediates germline development. Nature 450:1230-1234. doi:10.1038/nature06403

Chen PB, Chen HV, Acharya D, Rando OJ, Fazzio TG. 2015. R loops regulate promoterproximal chromatin architecture and cellular differentiation. Nat Struct Mol Biol 22:9991007. doi:10.1038/nsmb.3122

Chevrier S, Corcoran LM. 2014. BTB-ZF transcription factors, a growing family of regulators of early and late B-cell development. Immunol Cell Biol 92:481-488. doi:10.1038/icb.2014.20

Collins T, Stone JR, Williams AJ. 2001. All in the Family: the BTB/POZ, KRAB, and SCAN Domains. Mol Cell Biol 21:3609-3615. doi:10.1128/mcb.21.11.3609-3615.2001

Elling U, Taubenschmid J, Wirnsberger G, O'Malley R, Demers S-P, Vanhaelen Q, Shukalyuk Al, Schmauss G, Schramek D, Schnuetgen F, von Melchner H, Ecker JR, Stanford WL, Zuber J, Stark A, Penninger JM. 2011. Forward and Reverse Genetics through Derivation of Haploid Mouse Embryonic Stem Cells. Cell Stem Cell 9:563-574. doi:10.1016/j.stem.2011.10.012 
Elsässer SJ, Noh K-M, Diaz N, Allis CD, Banaszynski LA. 2015. Histone H3.3 is required for endogenous retroviral element silencing in embryonic stem cells. Nature 522:240-244. doi:10.1038/nature14345

Fazzio TG, Huff JT, Panning B. 2008. An RNAi Screen of Chromatin Proteins Identifies Tip60p400 as a Regulator of Embryonic Stem Cell Identity. Cell 134:162-174. doi:10.1016/j.cell.2008.05.031

Galonska C, Ziller MJ, Karnik R, Meissner A. 2015. Ground State Conditions Induce Rapid Reorganization of Core Pluripotency Factor Binding before Global Epigenetic Reprogramming. Cell Stem Cell 17:462-470. doi:10.1016/j.stem.2015.07.005

Goldberg AD, Banaszynski LA, Noh K-M, Lewis PW, Elsaesser SJ, Stadler S, Dewell S, Law M, Guo X, Li X, Wen D, Chapgier A, DeKelver RC, Miller JC, Lee Y-L, Boydston EA, Holmes MC, Gregory PD, Greally JM, Rafii S, Yang C, Scambler PJ, Garrick D, Gibbons RJ, Higgs DR, Cristea IM, Urnov FD, Zheng D, Allis CD. 2010. Distinct Factors Control Histone Variant H3.3 Localization at Specific Genomic Regions. Cell 140:678-691. doi:10.1016/j.cell.2010.01.003

Gontan C, Achame EM, Demmers J, Barakat TS, Rentmeester E, IJcken W van, Grootegoed JA, Gribnau J. 2012. RNF12 initiates X-chromosome inactivation by targeting REX1 for degradation. Nature 485:386-390. doi:10.1038/nature11070

Grover P, Asa JS, Campos El. 2018. H3-H4 Histone Chaperone Pathways. Annu Rev Genet 52:1-22. doi:10.1146/annurev-genet-120417-031547

Guezennec XL, Vermeulen M, Brinkman AB, Hoeijmakers WAM, Cohen A, Lasonder E, Stunnenberg HG. 2006. MBD2/NuRD and MBD3/NuRD, Two Distinct Complexes with Different Biochemical and Functional Properties. Mol Cell Biol 26:843-851. doi:10.1128/mcb.26.3.843-851.2006

Hayashi K, Ohta H, Kurimoto K, Aramaki S, Saitou M. 2011. Reconstitution of the Mouse Germ Cell Specification Pathway in Culture by Pluripotent Stem Cells. Cell 146:519-532. doi:10.1016/j.cell.2011.06.052

Helleboid P, Heusel M, Duc J, Piot C, Thorball CW, Coluccio A, Pontis J, Imbeault M, Turelli P, Aebersold R, Trono D. 2019. The interactome of KRAB zinc finger proteins reveals the evolutionary history of their functional diversification. Embo J 38:e101220. doi:10.15252/embj.2018101220

Hendrickson PG, Doráis JA, Grow EJ, Whiddon JL, Lim J-W, Wike CL, Weaver BD, Pflueger C, Emery BR, Wilcox AL, Nix DA, Peterson CM, Tapscott SJ, Carrell DT, Cairns BR. 2017. Conserved roles of mouse DUX and human DUX4 in activating cleavage-stage genes and MERVL/HERVL retrotransposons. Nat Genet 49:925-934. doi:10.1038/ng.3844

Ishiuchi T, Enriquez-Gasca R, Mizutani E, Bošković A, Ziegler-Birling C, Rodriguez-Terrones D, Wakayama T, Vaquerizas JM, Torres-Padilla M-E. 2015. Early embryonic-like cells are 
induced by downregulating replication-dependent chromatin assembly. Nat Struct Mol Biol 22:662-671. doi:10.1038/nsmb.3066

Jin C, Felsenfeld G. 2007. Nucleosome stability mediated by histone variants H3.3 and H2A.Z. Gene Dev 21:1519-1529. doi:10.1101/gad.1547707

Kaji K, Caballero IM, MacLeod R, Nichols J, Wilson VA, Hendrich B. 2006. The NuRD component $\mathrm{Mbd} 3$ is required for pluripotency of embryonic stem cells. Nat Cell Biol 8:285-292. doi:10.1038/ncb1372

Kaji K, Nichols J, Hendrich B. 2007. Mbd3, a component of the NuRD co-repressor complex, is required for development of pluripotent cells. Development 134:1123-1132. doi:10.1242/dev.02802

Kalkan T, Smith A. 2014. Mapping the route from naive pluripotency to lineage specification. Philosophical Transactions Royal Soc B Biological Sci 369:20130540. doi:10.1098/rstb.2013.0540

Karemaker ID, Vermeulen M. 2018. ZBTB2 reads unmethylated CpG island promoters and regulates embryonic stem cell differentiation. Embo Rep 19. doi:10.15252/embr.201744993

Kouhara H, Hadari YR, Spivak-Kroizman T, Schilling J, Bar-Sagi D, Lax I, Schlessinger J. 1997. A Lipid-Anchored Grb2-Binding Protein That Links FGF-Receptor Activation to the Ras/MAPK Signaling Pathway. Cell 89:693-702. doi:10.1016/s0092-8674(00)80252-4

Lambert SA, Jolma A, Campitelli LF, Das PK, Yin Y, Albu M, Chen X, Taipale J, Hughes TR, Weirauch MT. 2018. The Human Transcription Factors. Cell 172:650-665. doi:10.1016/j.cell.2018.01.029

Leeb M, Dietmann S, Paramor M, Niwa H, Smith A. 2014. Genetic Exploration of the Exit from Self-Renewal Using Haploid Embryonic Stem Cells. Cell Stem Cell 14:385-393. doi:10.1016/j.stem.2013.12.008

Leeb M, Pasini D, Novatchkova M, Jaritz M, Helin K, Wutz A. 2010. Polycomb complexes act redundantly to repress genomic repeats and genes. Gene Dev 24:265-276. doi:10.1101/gad.544410

Li T, Shi Y, Wang P, Guachalla LM, Sun B, Joerss T, Chen Y, Groth M, Krueger A, Platzer M, Yang Y, Rudolph KL, Wang Z. 2015. Smg6/Est1 licenses embryonic stem cell differentiation via nonsense-mediated mRNA decay. Embo J 34:1630-1647. doi:10.15252/embj.201489947

Lin T, Chao C, Saito S, Mazur SJ, Murphy ME, Appella E, Xu Y. 2005. p53 induces differentiation of mouse embryonic stem cells by suppressing Nanog expression. Nat Cell Biol 7:165-171. doi:10.1038/ncb1211 
Liu Z, Kraus WL. 2017. Catalytic-Independent Functions of PARP-1 Determine Sox2 Pioneer Activity at Intractable Genomic Loci. Mol Cell 65:589-603.e9. doi:10.1016/j.molcel.2017.01.017

Luck K, Kim D-K, Lambourne L, Spirohn K, Begg BE, Bian W, Brignall R, Cafarelli T, CamposLaborie FJ, Charloteaux B, Choi D, Coté AG, Daley M, Deimling S, Desbuleux A, Dricot A, Gebbia M, Hardy MF, Kishore N, Knapp JJ, Kovács IA, Lemmens I, Mee MW, Mellor JC, Pollis C, Pons C, Richardson AD, Schlabach S, Teeking B, Yadav A, Babor M, Balcha D, Basha O, Bowman-Colin C, Chin S-F, Choi SG, Colabella C, Coppin G, D'Amata C, Ridder DD, Rouck SD, Duran-Frigola M, Ennajdaoui H, Goebels F, Goehring L, Gopal A, Haddad G, Hatchi E, Helmy M, Jacob Y, Kassa Y, Landini S, Li R, Lieshout N van, MacWilliams A, Markey D, Paulson JN, Rangarajan S, Rasla J, Rayhan A, Rolland T, San-Miguel A, Shen Y, Sheykhkarimli D, Sheynkman GM, Simonovsky E, Taşan M, Tejeda A, Tropepe V, Twizere J-C, Wang Y, Weatheritt RJ, Weile J, Xia Y, Yang X, Yeger-Lotem E, Zhong Q, Aloy P, Bader GD, Rivas JDL, Gaudet S, Hao T, Rak J, Tavernier J, Hill DE, Vidal M, Roth FP, Calderwood MA. 2020. A reference map of the human binary protein interactome. Nature 580:402408. doi:10.1038/s41586-020-2188-x

Macfarlan TS, Gifford WD, Agarwal S, Driscoll S, Lettieri K, Wang J, Andrews SE, Franco L, Rosenfeld MG, Ren B, Pfaff SL. 2011. Endogenous retroviruses and neighboring genes are coordinately repressed by LSD1/KDM1A. Gene Dev 25:594-607. doi:10.1101/gad.2008511

Macfarlan TS, Gifford WD, Driscoll S, Lettieri K, Rowe HM, Bonanomi D, Firth A, Singer O, Trono D, Pfaff SL. 2012. Embryonic stem cell potency fluctuates with endogenous retrovirus activity. Nature 487:57-63. doi:10.1038/nature11244

Maeda T. 2016. Regulation of hematopoietic development by ZBTB transcription factors. Int J Hematol 104:310-323. doi:10.1007/s12185-016-2035-x

Martello G, Smith A. 2014. The Nature of Embryonic Stem Cells. Cell Dev Biology 30:647675. doi:10.1146/annurev-cellbio-100913-013116

Martello G, Sugimoto T, Diamanti E, Joshi A, Hannah R, Ohtsuka S, Göttgens B, Niwa H, Smith A. 2012. Esrrb Is a Pivotal Target of the Gsk3/Tcf3 Axis Regulating Embryonic Stem Cell Self-Renewal. Cell Stem Cell 11:491-504. doi:10.1016/j.stem.2012.06.008

Masuda T, Wang X, Maeda M, Canver MC, Sher F, Funnell APW, Fisher C, Suciu M, Martyn GE, Norton L, Zhu C, Kurita R, Nakamura Y, Xu J, Higgs DR, Crossley M, Bauer DE, Orkin SH, Kharchenko PV, Maeda T. 2016. Transcription factors LRF and BCL11A independently repress expression of fetal hemoglobin. Science 351:285-289. doi:10.1126/science.aad3312

Masui S, Ohtsuka S, Yagi R, Takahashi K, Ko MS, Niwa H. 2008. Rex1/Zfp42 is dispensable for pluripotency in mouse ES cells. Bmc Dev Biol 8:45. doi:10.1186/1471-213x-8-45

Mattout A, Aaronson Y, Sailaja BS, Ram EVR, Harikumar A, Mallm J-P, Sim KH, Nissim-Rafinia M, Supper E, Singh PB, Sze SK, Gasser SM, Rippe K, Meshorer E. 2015. Heterochromatin 
Protein $1 \beta$ (HP1 $\beta$ ) has distinct functions and distinct nuclear distribution in pluripotent versus differentiated cells. Genome Biol 16:213. doi:10.1186/s13059-015-0760-8

Mena EL, Kjolby RAS, Saxton RA, Werner A, Lew BG, Boyle JM, Harland R, Rape M. 2018. Dimerization quality control ensures neuronal development and survival. Science 362:eaap8236. doi:10.1126/science.aap8236

Molotkov A, Mazot P, Brewer JR, Cinalli RM, Soriano P. 2017. Distinct Requirements for FGFR1 and FGFR2 in Primitive Endoderm Development and Exit from Pluripotency. Dev Cell 41:511-526.e4. doi:10.1016/j.devcel.2017.05.004

Pradhan SK, Su T, Yen L, Jacquet K, Huang C, Côté J, Kurdistani SK, Carey MF. 2016. EP400 Deposits H3.3 into Promoters and Enhancers during Gene Activation. Mol Cell 61:27-38. doi:10.1016/j.molcel.2015.10.039

Ray-Gallet D, Woolfe A, Vassias I, Pellentz C, Lacoste N, Puri A, Schultz DC, Pchelintsev NA, Adams PD, Jansen LET, Almouzni G. 2011. Dynamics of Histone H3 Deposition In Vivo Reveal a Nucleosome Gap-Filling Mechanism for H3.3 to Maintain Chromatin Integrity. Mol Cell 44:928-941. doi:10.1016/j.molcel.2011.12.006

Reynolds N, Latos P, Hynes-Allen A, Loos R, Leaford D, O'Shaughnessy A, Mosaku O, Signolet J, Brennecke P, Kalkan T, Costello I, Humphreys P, Mansfield W, Nakagawa K, Strouboulis J, Behrens A, Bertone P, Hendrich B. 2012. NuRD Suppresses Pluripotency Gene Expression to Promote Transcriptional Heterogeneity and Lineage Commitment. Cell Stem Cell 10:583-594. doi:10.1016/j.stem.2012.02.020

Rodriguez-Terrones D, Gaume X, Ishiuchi T, Weiss A, Kopp A, Kruse K, Penning A, Vaquerizas JM, Brino L, Torres-Padilla M-E. 2018. A molecular roadmap for the emergence of earlyembryonic-like cells in culture. Nat Genet 50:106-119. doi:10.1038/s41588-017-0016-5

Sadic D, Schmidt K, Groh S, Kondofersky I, Ellwart J, Fuchs C, Theis FJ, Schotta G. 2015. Atrx promotes heterochromatin formation at retrotransposons. Embo Rep 16:836-850. doi:10.15252/embr.201439937

Sarai N, Nimura K, Tamura T, Kanno T, Patel MC, Heightman TD, Ura K, Ozato K. 2013. WHSC1 links transcription elongation to HIRA-mediated histone H3.3 deposition. Embo J 32:2392-2406. doi:10.1038/emboj.2013.176

Sato N, Meijer L, Skaltsounis L, Greengard P, Brivanlou AH. 2004. Maintenance of pluripotency in human and mouse embryonic stem cells through activation of Wnt signaling by a pharmacological GSK-3-specific inhibitor. Nat Med 10:55-63. doi:10.1038/nm979

Schnütgen F, Hansen J, De-Zolt S, Horn C, Lutz M, Floss T, Wurst W, Noppinger PR, Melchner $\mathrm{H}$ von. 2008. Enhanced gene trapping in mouse embryonic stem cells. Nucleic Acids Res 36:e133-e133. doi:10.1093/nar/gkn603 
Scotland KB, Chen S, Sylvester R, Gudas LJ. 2009. Analysis of Rex1 (zfp42) function in embryonic stem cell differentiation. Dev Dynam 238:1863-1877. doi:10.1002/dvdy.22037

Siggs O, Beutler B. 2012. The BTB-ZF transcription factors. Cell Cycle 11:3358-3369. doi:10.4161/cc.21277

Silva J, Smith A. 2008. Capturing Pluripotency. Cell 132:532-536. doi:10.1016/j.cell.2008.02.006

Smith AG, Heath JK, Donaldson DD, Wong GG, Moreau J, Stahl M, Rogers D. 1988. Inhibition of pluripotential embryonic stem cell differentiation by purified polypeptides. Nature 336:688-690. doi:10.1038/336688a0

Stogios PJ, Downs GS, Jauhal JJ, Nandra SK, Privé GG. 2005. Sequence and structural analysis of BTB domain proteins. Genome Biol 6:R82. doi:10.1186/gb-2005-6-10-r82

Tagami H, Ray-Gallet D, Almouzni G, Nakatani Y. 2004. Histone H3.1 and H3.3 Complexes Mediate Nucleosome Assembly Pathways Dependent or Independent of DNA Synthesis. Cell 116:51-61. doi:10.1016/s0092-8674(03)01064-x

Tee W-W, Shen SS, Oksuz O, Narendra V, Reinberg D. 2014. Erk1/2 Activity Promotes Chromatin Features and RNAPII Phosphorylation at Developmental Promoters in Mouse ESCs. Cell 156:678-690. doi:10.1016/j.cell.2014.01.009

Thinon E, Serwa RA, Broncel M, Brannigan JA, Brassat U, Wright MH, Heal WP, Wilkinson AJ, Mann DJ, Tate EW. 2014. Global profiling of co- and post-translationally N-myristoylated proteomes in human cells. Nat Commun 5:4919. doi:10.1038/ncomms5919

Villegas F, Lehalle D, Mayer D, Rittirsch M, Stadler MB, Zinner M, Olivieri D, Vabres P, Duplomb-Jego L, Bont ESJMD, Duffourd Y, Duijkers F, Avila M, Geneviève D, Houcinat N, Jouan T, Kuentz P, Lichtenbelt KD, Thauvin-Robinet C, St-Onge J, Thevenon J, Gassen KLI van, Haelst $M$ van, Koningsbruggen $S$ van, Hess $D$, Smallwood SA, Rivière J-B, Faivre $L$, Betschinger J. 2019. Lysosomal Signaling Licenses Embryonic Stem Cell Differentiation via Inactivation of Tfe3. Cell Stem Cell 24:257-270.e8. doi:10.1016/j.stem.2018.11.021

Weirauch MT, Yang A, Albu M, Cote AG, Montenegro-Montero A, Drewe P, Najafabadi HS, Lambert SA, Mann I, Cook K, Zheng H, Goity A, van Bakel H, Lozano J-C, Galli M, Lewsey MG, Huang E, Mukherjee T, Chen X, Reece-Hoyes JS, Govindarajan S, Shaulsky G, Walhout AJM, Bouget F-Y, Ratsch G, Larrondo LF, Ecker JR, Hughes TR. 2014. Determination and Inference of Eukaryotic Transcription Factor Sequence Specificity. Cell 158:1431-1443. doi:10.1016/j.cell.2014.08.009

Wray J, Kalkan T, Gomez-Lopez S, Eckardt D, Cook A, Kemler R, Smith A. 2011. Inhibition of glycogen synthase kinase-3 alleviates Tcf3 repression of the pluripotency network and increases embryonic stem cell resistance to differentiation. Nat Cell Biol 13:838-845. doi:10.1038/ncb2267 
Wray J, Kalkan T, Smith AG. 2010. The ground state of pluripotency. Biochem Soc T 38:10271032. doi:10.1042/bst0381027

Xiong C, Wen Z, Yu J, Chen J, Liu C-P, Zhang X, Chen P, Xu R-M, Li G. 2018. UBN1/2 of HIRA complex is responsible for recognition and deposition of $\mathrm{H} 3.3$ at cis-regulatory elements of genes in mouse ES cells. Bmc Biol 16:110. doi:10.1186/s12915-018-0573-9

Xue Y, Wong J, Moreno GT, Young MK, Côté J, Wang W. 1998. NURD, a Novel Complex with Both ATP-Dependent Chromatin-Remodeling and Histone Deacetylase Activities. Mol Cell 2:851-861. doi:10.1016/s1097-2765(00)80299-3

Yang S-H, Kalkan T, Morrisroe C, Smith A, Sharrocks AD. 2012. A Genome-Wide RNAi Screen Reveals MAP Kinase Phosphatases as Key ERK Pathway Regulators during Embryonic Stem Cell Differentiation. Plos Genet 8:e1003112. doi:10.1371/journal.pgen.1003112

Yang SH, Shrivastav A, Kosinski C, Sharma RK, Chen M-H, Berthiaume LG, Peters LL, Chuang P-T, Young SG, Bergo MO. 2005. N-Myristoyltransferase 1 Is Essential in Early Mouse Development. J Biol Chem 280:18990-18995. doi:10.1074/jbc.m412917200

Ying Q-L, Wray J, Nichols J, Batlle-Morera L, Doble B, Woodgett J, Cohen P, Smith A. 2008. The ground state of embryonic stem cell self-renewal. Nature 453:519-523. doi:10.1038/nature06968

Youn H-D, Sun L, Prywes R, Liu JO. 1999. Apoptosis of T Cells Mediated by Ca2+-Induced Release of the Transcription Factor MEF2. Science 286:790-793. doi:10.1126/science.286.5440.790 


\section{Genetic screen}

$175^{*} 10^{6}$ haploid mESCs B1A4 Oct4>GFP-Puro (Elling et al., 2011) were transduced with each of the retroviruses reFlipROSAßgeo(Cre)*0, reFlipROSAßgeo(Cre $)^{*}+1$, reFlipROSAßgeo(Cre)*+2, rsFlipROSAßgeo*+2 (Schnütgen et al., 2008), selected with Neomycin, and frozen. After thawing, cells were allowed to recover in Serum-LIF (GMEM (Sigma), 10\% fetal bovine serum (Sigma), 1mM sodium pyruvate (Gibco), $2 \mathrm{mM}$ L-glutamine (Gibco), 0.1mM non-essential amino acids (Gibco), 0.1mM 2-mercaptoethanol (Sigma), and $1000 \mathrm{U} / \mathrm{mL} \mathrm{mLIF}$ (Chao lab, Basel)) for 24 hours and then transferred on day 0 to N2B27 (DMEM/F12 medium (Life Technologies) and Neurobasal medium (Gibco) 1:1, N2 supplement 1/200 (homemade), B-27 Serum-Free Supplement 1/100 (Gibco), 2mM L-glutamine (Gibco),

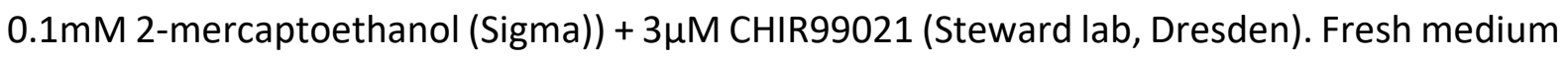
was provided every 2 days and the cells were replated every 4 days until day 13 , when the medium was changed to N2B27 + CHIR + Puromycin. Half of the cells were harvested on day 16 , then again on day 20 , and finally all on day 23 . Cell pellets were digested overnight at $56^{\circ} \mathrm{C}$ in $10 \mathrm{mM}$ Tris $\mathrm{HCl}$ pH7.5, $10 \mathrm{mM}$ EDTA, $10 \mathrm{mM} \mathrm{NaCl}, 0.5 \% \mathrm{SDS}, 1 \mathrm{mg} / \mathrm{mL}$ proteinase $\mathrm{K}$ (MachereyNagel) and then with $0.1 \mathrm{mg} / \mathrm{mL}$ RNaseA (Qiagen) for $2 \mathrm{~h}$ at $37^{\circ} \mathrm{C}$. DNA was ethanol precipitated, washed twice with ethanol $70 \%$, and resuspended in $\mathrm{H}_{2} \mathrm{O}$. $5 \mathrm{ug}$ of DNA were digested with Dpnll or Msel (NEB) for $5 \mathrm{~h}$ at $37^{\circ} \mathrm{C}$, purified with the PCR cleanup kit (Qiagen), ligated in $250 \mu \mathrm{L}$ with $3 \mu \mathrm{L}$ of T4 ligase (NEB) at $16^{\circ} \mathrm{C}$ for $36 \mathrm{~h}$, and purified with the PCR cleanup kit (Qiagen). Half of the eluate was redigested with Nhel and half with Pvull (NEB), the reactions were pooled and purified by PCR cleanup kit (Qiagen) eluted in $50 \mu \mathrm{L}$. $10 \mu \mathrm{L}$ of eluate were used as a template for PCR with KOD (Takara) with extension time 1'10', annealing temperature $58^{\circ} \mathrm{C}$, for 35 cycles, with primers aatgatacggcgaccaccgagatctacacGCCAGTCCTCCGATTGA and caagcagaagacggcatacgagatBBBBBBAGTTCCTATTCCGAAGTTCCTATTCTCTA (B=barcode). PCRs were purified with the PCR cleanup kit (Qiagen), and subjected to next generation sequencing with sequencing primer TGATTGACTACCCGTCAGCGGGGGTCTTTCA and indexing primer TATACTTTCTAG+A+GAATAGGAACTTCGGAATA+G+GAACT $\quad(+\mathrm{N}=$ LNA modification).

Sequence reads were processed to remove adaptors (Msel TTAA and DpnII GATC) and then mapped to the mouse reference genome ( $\mathrm{mm} 9$ only chromosomes 1 to $19, \mathrm{X}, \mathrm{Y}$ and $\mathrm{M}$ ) using Bowtie version 1.0.0 with parameters - $\mathrm{v} 3-\mathrm{m} 1$ - best - strata. Output SAM files were sorted and converted to BAM files using samtools version $0.1 .19-44428 \mathrm{~cd}$. Genomic tracks were generated using genomeCoverageBed from bedtools version 2.25.0 using only the first base pair position of each read according to the strand. The number of insertions per gene (using only insertions supported by more than one read) normalized to 10,000 reads per library was generated using the ENSEMBL gtf annotation Mus_musculus.NCBIM37.67. Most of this analysis was processed using the unix command awk. The $\mathrm{R}$ version 3.3.2 was used to compute a mean log2 fold change (using a pseudocount of 0.5 ) contrasting each group to the corresponding control and a z-score within each library (Table S1). 


\section{Cell culture}

TNG-A mESCs (Chambers et al., 2007) were cultured in 2iLIF (N2B27, 1 $\mu \mathrm{M}$ PD0325901 (Steward lab, Dresden), $3 \mu \mathrm{M}$ CHIR99021, and 1000U/mL mLIF) on gelatin-coated tissue culture plates. For medium switch experiments, cells were washed with PBS, detached with Accutase (Sigma), centrifuged for 3' at 300g in DMEM/F12-0.1\% BSA, resuspended in the new medium, counted, and replated. In experiments with doxycycline inducible constructs, $1 \mu \mathrm{g} / \mathrm{mL}$ doxycycline (Sigma) was added to the final medium. Cells were transitioned to N2B27 $+3 \mu \mathrm{M}$ CHIR99021 + $12 \mathrm{ng} / \mathrm{ml}$ bFGF (Smith lab, Cambridge) on gelatin coated plates at a density of $2^{\prime} 500 / \mathrm{cm}^{2}$, or to EpiLC medium ((N2B27 base, $20 \mathrm{ng} / \mathrm{ml}$ activin A, $12 \mathrm{ng} / \mathrm{ml} \mathrm{bFGF}$ (Smith lab, Cambridge), and 1\% KSR (Life Technologies)) on fibronectin coated plates at a density of $25^{\prime} 000 / \mathrm{cm}^{2}$, or to Serum-LIF on gelatin coated plates at a density of $2^{\prime} 500 / \mathrm{cm}^{2}$. At the moment of analysis, cells were washed with PBS, resuspended with trypsin (Life Technologies), centrifuged for $3^{\prime}$ at $300 \mathrm{~g}$ in DMEM/F12-0.1\% BSA, resuspended in DMEM/F12-0.1\% BSA, and flowed on an LSRII SORP Analyzer (Becton Dickinson). Percentage of GFP-high cells was quantified with BD FACSDiva 8.0.1 and flow profiles were made with FlowJo (FlowJo, LLC).

\section{Mutant cell lines and overexpression constructs}

A TNG-A clone stably expressing Cas9 (TbC1) was derived by transfection of PB-LR5.1-EF1absdr2ACas9 (derived from pPB-LR5.1-EF1a-puro2ACas9, gift of Kosuke Yusa, Wellcome Trust Sanger Institute) and PBase (PiggyBac Transposase, (Betschinger et al., 2013)). To generate $\mathrm{KO}$ cell lines, TbC1 cells were reverse transfected with 400ng of U6>sgRNA plasmids (George Church, Addgene plasmid \#41824) according to Table S2 and 3 $\mu$ L Lipofectamin 2000 (Life Technologies). Single cells were sorted in 96-well plates in 2iLIF and expanded. For constitutive or inducible expression, the cDNA of the gene of interest was cloned in PPB-CAGDEST-pgk-hph (CAG>) (Betschinger et al., 2013) or pPB-TRE-DEST-rTA-HSV-neo (DOX>) (Villegas et al., 2019), respectively, and $1 \mu \mathrm{g}$ plasmid was reverse-transfected together with $1 \mu \mathrm{g}$ PBase and $3 \mu \mathrm{L}$ Lipofectamin 2000 in TbC1 cells. The next day fresh medium with the appropriate selection was added and the cells were analyzed after at least 1 week of selection.

\section{Immunofluorescence}

Cells were plated on a laminin-coated 96-well glass plate (Greiner Bio-One), fixed with PBS4\% PFA for 20', washed twice with PBS, permeabilized with PBS-0.1\% Triton X-100 for 10' at room temperature (RT), incubated in blocking solution (3\% donkey serum (Sigma), 1\% BSA in PBS-0.1\% Tween-20 (PBST)) for $1 \mathrm{~h}$ at RT, incubated with anti-Tfe3 antibody (Sigma, Cat\# HPA023881, RRID:AB_1857931) 1/1000 in blocking solution overnight at $4^{\circ} \mathrm{C}$, washed three times with PBST, stained for $2 \mathrm{~h}$ at RT with secondary antibody Donkey Anti-Rabbit IgG - Alexa Fluor 555 (Life Technologies) 1/500 in PBST, counterstained with PBST-Hoechst33342 1/5000 (Life Technologies), washed twice with PBS and imaged at an LSM-710 scanning head confocal microscope (Zeiss). Images were exclusively cropped with no further manipulation. 
WT and $\mathrm{Nmt1}^{-/-}$mESCs grown in 2iLIF were washed twice with PBS and incubated for $30^{\prime}$ or $6 \mathrm{~h}$ in N2B27 $+12 \mathrm{ng} / \mathrm{ml}$ bFGF, put on ice, harvested, and lysed in RIPA buffer (50mM Tris pH7.5, 150mM NaCl, 1\% IGEPAL, 0.5\% Na Deoxycholate, 0.1\% SDS, 2mM EDTA) with fresh Complete Protease Inhibitor Tablet (Roche) and Phosphatase Inhibitor Tablet (Roche). 10 $\mathrm{gg}$ of cell lysate were resolved on a 10\% SDS-PAGE and wet-blotted on nitrocellulose. Separate membranes were probed with Erk1/2 antibody (Cell Signaling Technology, \#9102) or Phospho-Erk1/2 antibody (Cell Signaling Technology, \#9101) 1/1000 in PBST-5\% BSA.

\section{Affinity-purification mass-spectrometry}

$2 * 10^{5}$ WT or mutant naïve TNG-A cells were transfected in triplicate with $1 \mu \mathrm{g}$ pgk>BirA plasmid and $1 \mu \mathrm{g}$ DOX>prey plasmid according to Table S4 or no plasmid as negative control with $3 \mu \mathrm{L}$ Lipofectamin 2000 . The next day fresh medium with hygromycin (selection for the pgk>BirA plasmid) was applied. Cells were grown in selective medium for 3-4 days and then the medium was changed to Serum-LIF $+1 \mu \mathrm{g} / \mathrm{mL}$ Doxycycline. After 48 hours $10^{7}$ cells were harvested with trypsin, washed in PBS-0.1\%BSA, washed in PBS, and nuclei were extracted in $10 \mathrm{mM}$ Tris $\mathrm{HCl}$ pH7.5, $10 \mathrm{mM} \mathrm{KCl}, 1 \mathrm{mM}$ DTT, $0.5 \%$ IGEPAL, with Complete protease inhibitor (Roche) for $20^{\prime}$ minutes on ice. Nuclei were lysed by rotation for $1 \mathrm{~h}$ at $4^{\circ} \mathrm{C}$ in $20 \mathrm{mM} \mathrm{Tris} \mathrm{HCl}$ $7.5,100 \mathrm{mMKCl}, 1.5 \mathrm{mM} \mathrm{MgCl}_{2}, 1 \mathrm{mM}$ DTT, $10 \%$ glycerol, $0.5 \%$ TritonX-100, Complete protease inhibitor (Roche), Phosphatase inhibitor (Roche), and $250 \mathrm{U} / \mathrm{mL}$ Benzonase (Merck). Lysates were clarified by centrifugation for $5^{\prime}$ at $12^{\prime} 000 \mathrm{~g}$ at $4^{\circ} \mathrm{C}, 10 \mu \mathrm{L}$ of $\mathrm{M} 280$ StreptavidinDynabeads (ThermoFisher) were added and incubated at $4^{\circ} \mathrm{C}$ rotating for $4 \mathrm{~h}$. Beads were then washed three times with $20 \mathrm{mM}$ Tris $\mathrm{HCl}$ pH7.5, $150 \mathrm{mM} \mathrm{NaCl}$, with $0.5 \%$ IGEPAL and twice without IGEPAL. Beads were digested with Lys-C at RT for $4 \mathrm{~h}$ and then with trypsin overnight at $37^{\circ} \mathrm{C}$.

The generated peptides were acidified with TFA to a final concentration of $0.8 \%$ and analyzed by capillary liquid chromatography tandem mass spectrometry with an EASY-nLC 1000 using the two-column set-up (Thermo Scientific). The peptides were loaded with $0.1 \%$ formic acid, $2 \%$ acetonitrile in $\mathrm{H}_{2} \mathrm{O}$ onto a peptide trap (Acclaim PepMap 100, 75um $\times 2 \mathrm{~cm}, \mathrm{C} 18,3 \mathrm{um}$, $100 \AA$ ) at a constant pressure of 800 bar. Peptides were separated, at a flow rate of $150 \mathrm{~nL} / \mathrm{min}$ with a linear gradient of $2-6 \%$ buffer $B$ in buffer $A$ in 3 minutes followed by an linear increase from 6 to $22 \%$ in 40 minutes, $22-28 \%$ in $9 \mathrm{~min}, 28-36 \%$ in $8 \mathrm{~min}, 36-80 \%$ in $1 \mathrm{~min}$ and the column was finally washed for $14 \mathrm{~min}$ at $80 \%$ B (Buffer A: $0.1 \%$ formic acid, buffer B: $0.1 \%$ formic acid in acetonitrile) on a $50 \mu \mathrm{m} \times 15 \mathrm{~cm}$ ES801 C18, $2 \mu \mathrm{m}, 100 \AA$ column (Thermo Scientific) mounted on a DPV ion source (New Objective) connected to a Orbitrap Fusion (Thermo Scientific). The data were acquired using 120000 resolution for the peptide measurements in the Orbitrap and a top T (3s) method with HCD fragmentation for each precursor and fragment measurement in the ion trap according the recommendation of the manufacturer (Thermo Scientific). Protein identification and relative quantification of the proteins was done with MaxQuant version 1.5.3.8 using Andromeda as search engine (Cox et al., 2011) and label free quantification (LFQ, (Cox et al., 2014)) as described (Hubner et al., 2010). The mouse subset of the UniProt data base combined with the contaminant database from MaxQuant was searched and the protein and peptide FDR were set to 0.01 . 
The LFQ values were analyzed with Perseus v.1.6.2.2 as follows: entries identified only by site or reverse and potential contaminants were removed, values were Log2 transformed, entries identified in less than 2 replicates in any group were removed, and missing values were imputed based on the normal distribution of each replicate with a 0.25 -fold width and a down-shift of 1.8-fold. Volcano plots are based on 2-sided t-test and threshold curves on an SO $=0.1$ and FDR=0.0054 for Zbtb2 baits and FDR=0.02 for Ubn1/2 baits.

For the heatmap representation in Figure 2B, missing Mascot values in experimental triplicates of Zbtb2 mutant AP-MS were imputed with a 1.8-fold downshift and a 0.25 -fold distribution width of the actual distribution of detected proteins using the fitdistr function from the Cran package MASS (https://cran.r-project.org/web/packages/MASS/index.html), as described (Tyanova et al., 2016). To correct for varying Zbtb2 amounts in different APs, protein enrichments in each AP were normalized to the respective Zbtb2 bait. To compare interaction strengths, the enrichment of each interactor was normalized to its enrichment in wildtype Zbtb2 purifications.

\section{Yeast-2-hybrid}

Yeast-2-hybrid assays were performed with the plasmids and strains from the Matchmaker Gold Yeast Two-Hybrid System (Takara Bio) according to manufacturer's protocol, with the following plasmid modifications. For N-terminal AD- fusions, pGADT7 was digested with Ndel and BamHI and religated with oligo TAGTGGTGGAACAAAAATGGGCCCGAATTCCCGGGATCGATTAACTGAGTAG, to create Apal and Clal sites for InFusion cloning (Takara Bio). For C-terminal -AD and -DBD fusions, TTTAAACTATTTGGGCCCATTTTTGTTCCACCACTATAAGCTTGGAGTTGATTGTATGCTTGG and either

\section{AAATGGGCCCAAATAGTTTAAACCGCGGTGGATCTGGTGGAATGGATAAAGCGGAATTAATTCCCG} AG

AAATGGGCCCAAATAGTTTAAACCGCGGTGGATCTGGTGGAATGAAGCTACTGTCTTCTATCGAACA $A G C$ were used for site-directed mutagenesis of pGADT7 and pGBKT7, respectively, to create a new MCS with Apal and Sacll sites for InFusion cloning (Takara Bio). pGBKT7-C and pGADT7$C$ were digested with $\mathrm{Ndel}$ and BamHI and religated with the oligo TATGCCAGCTGCTAAAAGAGTTAAATTGGATTAG to create a new c-Myc NLS. Briefly, pGBKT7 and pGADT7 plasmids were transformed into the yeast strains $\mathrm{Y} 2 \mathrm{HGold}$ and $\mathrm{Y} 187$, respectively. Several colonies were picked and grown overnight in SD-Trp or SD-Leu, for pGBKT7 or pGADT7 plasmids respectively. When the cultures reached an OD600 0.5, they were mated overnight in YPD medium and grown on an SC-Trp/-Leu plate for 2-3 days, and plate replicas were made on an SD-Ade/-His/-Trp/-Leu/+X-alpha-Gal/+Aureobasidin A plate. Pictures of the plates were taken after 2-4 days and images were exclusively cropped, with no further processing.

\section{Phylogeny}

Btb domain sequences were retrieved with the 'Architecture analysis' tool of SMART (smart.embl.de; query: "BTB AND ZnF_C2H2" in "Mus musculus") or individually with the 'Sequence analysis' tool. The phylogenetic tree was calculated, based on a multiple sequence alignment generated with T-Coffee (https://www.ebi.ac.uk/Tools/msa/tcoffee/), using the neighbour-joining clustering method provided by the ClustalW2 package 
1 (http://www.clustal.org/clustal2/) running 1000 iterations. The tree was visualized with iTOL

2 (https://itol.embl.de/).

\section{Gene expression analysis}

RNAseq reads and published data (see table below) were aligned to the mouse $\mathrm{GRCm} 38 / \mathrm{mm} 10$ genome using qAlign from the Bioconductor package QuasR (Gaidatzis et al., 2015) with default parameters except for aligner="Rhisat2" and splicedAlignment=TRUE. For aligning RNAseq reads for Dux overexpression (Hendrickson et al., 2017), paired=" $f r$ " was additionally used. Alignments were quantified for known UCSC genes obtained from the TxDb.Mmusculus.UCSC.mm10.knownGene package using qCount. Microarray data (Fazzio et al., 2008) was analyzed and normalized using the Bioconductor package limma (Ritchie et al., 2015). In this dataset, Tip60 knockdown replicate 3 is an outlier, and Ep400 knockdown replicates 1 and 3 do not show Ep400 transcript reduction, and were therefore excluded from the analysis.

Differential gene expression (Table S3) was determined using edgeR (Robinson and Oshlack, 2010). In Fig. 1G, Fig. S1G, and Fig. S5A preEPI is the combination of the EPI and ICM+EPI markers, and postEPI the PE geneset (Boroviak et al., 2015). Pearson correlation coefficients (Fig. 5C) were calculated using R's cor function. For heatmap visualization (Fig. 5D), significantly deregulated genes in Zbtb2 mutants were considered (Table S3), which are the genes that showed an absolute log2 expression FC of greater than 1 with a false discovery rate of less than 0.005 between wildtype and $\mathrm{Zbtb} 2 \mathrm{KO}$ cells in at least one of the conditions: 2iLIF, 24h EpiLC, 48h EpiLC, 24h Serum-LIF, 48h Serum-LIF. All contrast shown in Fig. 5D were used for clustering.

\begin{tabular}{|l|l|l|}
\hline Accession & Description & Reference \\
\hline GSE85505 & Kat5 knockdown, RNA-seq & (Acharya et al., 2017) \\
\hline E-MTAB-997 & Mbd3 knockout, RNA-seq & (Reynolds et al., 2012) \\
\hline GSE85632 & DUX overexpression 24h, RNA-seq & (Hendrickson et al., 2017) \\
\hline GSE11243 & Kat5 and Ep400 knockdown, microarray & (Fazzio et al., 2008) \\
\hline
\end{tabular}

24

\section{ChIPseq analysis}

Published datasets (see table below) were aligned to the mouse GRCm38/mm10 genome using qAlign and profiled using qProfile from the Bioconductor package QuasR. 137435 ATAC peaks (Olivieri et al., 2020) were called using Macs2 (Zhang et al., 2008), of which 22826 were in promoters (+/- $1 \mathrm{~kb}$ of annotated transcriptional start sites). For heatmap visualization (Fig. S5D), ChIPseq signals were profiled in these promoters and ChIP enrichment calculated over respective inputs (Zbtb2, H3K4me3, Chd4, Mbd3, Dux) or controls (Kat5, Ep400). For metaplots (Fig. 5E, F, Fig. S5B, C), ChIPseq signals were profiled in promoter regions of cluster genes that were extracted from the TxDb.Mmusculus.UCSC.mm10.knownGene Bioconductor package

(https://bioconductor.org/packages/release/data/annotation/html/TxDb.Mmusculus.UCSC. mm10.knownGene.html). 


\begin{tabular}{|l|l|l|}
\hline Accession & Description & Reference \\
\hline GSE85632 & DUX 18h, ChIP-seq & (Hendrickson et al., 2017) \\
\hline GSE101802 & Zbtb2 2i, ChIP-seq & (Karemaker and Vermeulen, 2018) \\
\hline E-MTAB-6804 & Mbd3 and Chd4, ChIP-seq & (Bornelöv et al., 2018) \\
\hline GSE67584 & Kat5 and Ep400, ChIP-seq & (Chen et al., 2015) \\
\hline GSE74112 & K3K4me3, ChIP-seq & (Liu and Kraus, 2017) \\
\hline E-MTAB-9453 & ATAC-seq & (Olivieri et al., 2020) \\
\hline
\end{tabular}

1

\section{$R T-q P C R$}

RNA was extracted from naïve mESCs with the RNeasy Mini Kit (Qiagen) and $1 \mu \mathrm{g}$ of it was reverse-transcribed with SuperScript III Reverse Transcriptase (LifeTechnologies). qPCR was performed with the TaqMan Fast Universal PCR Master Mix (ThermoFisher) and the TaqMan probes GAPDH (4352339E) and Zbtb2 (Mm01605943_g1). The DOX>Zbtb2 plasmid that cannot be detected by Mm01605943_g1 was produced by fusion PCR of the endogenous cDNA and the following gBlock (Integrated DNA Technologies): ATGGATTTGACCAACCATGGACTTATTCTACTGCAGCAGTTAAACGCTCAGCGAGAGTTTGGTTTCCT GTGTGACTGCACGGTTGCAATCGGCGATGTGTATTTTAAAGCCCATAAGAGTGTGTTGGCAAGTTTT AGTAACTATTTCAAAATGCTTTTCGTGCACCAAACATCAGAGTGTGTGAGATTAAAACCAACAGATA TCCAACCAGATATCTTTTCATACTTATTGCATTTAATGTATACCGGGAAGATGGCCCCACAGCTCATC GACCCTGTGAGGCTAGAGCAAGGGATCAAATTCCTGCACGCATACCCCCTCATCCAGGAAGCCAGC CTTGCCAGCCAAGGCAGCTTTTCCCATCCCGAGCAAGTCTTCCCTCTGGCCTCATCCTTGTACGGCAT TCAGATTGCAGACCATCAGCTGAGACAAGCCACCAAGATGAATTTAGGGCCTGAGAAACTTGGACG GGAGCCTAGGCCACAGGCATCCAGGATGA. The construct was validated by plasmid qPCR with Mm01605943_g1.

\section{Data availability}

The genomic data generated for this study have been deposited at ArrayExpress (E-MTAB9796, E-MTAB-9797, E-MTAB-9798). The mass spectrometry proteomics data have been deposited at the ProteomeXchange Consortium via the PRIDE partner repository with the dataset identifiers PXD022451 and PXD022446.

\section{Materials and methods references}

Acharya D, Hainer SJ, Yoon Y, Wang F, Bach I, Rivera-Pérez JA, Fazzio TG. 2017. KATIndependent Gene Regulation by Tip60 Promotes ESC Self-Renewal but Not Pluripotency. Cell Reports 19:671-679. doi:10.1016/j.celrep.2017.04.001

Betschinger J, Nichols J, Dietmann S, Corrin PD, Paddison PJ, Smith A. 2013. Exit from Pluripotency Is Gated by Intracellular Redistribution of the bHLH Transcription Factor Tfe3. Cell 153:335-347. doi:10.1016/j.cell.2013.03.012 
Bornelöv S, Reynolds N, Xenophontos M, Gharbi S, Johnstone E, Floyd R, Ralser M, Signolet J, Loos R, Dietmann S, Bertone P, Hendrich B. 2018. The Nucleosome Remodeling and Deacetylation Complex Modulates Chromatin Structure at Sites of Active Transcription to Fine-Tune Gene Expression. Mol Cell 71:56-72.e4. doi:10.1016/j.molcel.2018.06.003

Boroviak T, Loos R, Lombard P, Okahara J, Behr R, Sasaki E, Nichols J, Smith A, Bertone P. 2015. Lineage-Specific Profiling Delineates the Emergence and Progression of Naive Pluripotency in Mammalian Embryogenesis. Dev Cell 35:366-382. doi:10.1016/j.devcel.2015.10.011

Chambers I, Silva J, Colby D, Nichols J, Nijmeijer B, Robertson M, Vrana J, Jones K, Grotewold L, Smith A. 2007. Nanog safeguards pluripotency and mediates germline development. Nature 450:1230-1234. doi:10.1038/nature06403

Chen PB, Chen HV, Acharya D, Rando OJ, Fazzio TG. 2015. R loops regulate promoter-proximal chromatin architecture and cellular differentiation. Nat Struct Mol Biol 22:999-1007. doi:10.1038/nsmb.3122

Cox J, Hein MY, Luber CA, Paron I, Nagaraj N, Mann M. 2014. Accurate Proteome-wide Labelfree Quantification by Delayed Normalization and Maximal Peptide Ratio Extraction, Termed MaxLFQ. Mol Cell Proteomics 13:2513-2526. doi:10.1074/mcp.m113.031591

Cox J, Neuhauser N, Michalski A, Scheltema RA, Olsen JV, Mann M. 2011. Andromeda: A Peptide Search Engine Integrated into the MaxQuant Environment. J Proteome Res 10:1794-1805. doi:10.1021/pr101065j

Elling U, Taubenschmid J, Wirnsberger G, O'Malley R, Demers S-P, Vanhaelen Q, Shukalyuk Al, Schmauss G, Schramek D, Schnuetgen F, von Melchner H, Ecker JR, Stanford WL, Zuber J, Stark A, Penninger JM. 2011. Forward and Reverse Genetics through Derivation of Haploid Mouse Embryonic Stem Cells. Cell Stem Cell 9:563-574. doi:10.1016/j.stem.2011.10.012

Fazzio TG, Huff JT, Panning B. 2008. An RNAi Screen of Chromatin Proteins Identifies Tip60p400 as a Regulator of Embryonic Stem Cell Identity. Cell 134:162-174. doi:10.1016/j.cell.2008.05.031

Gaidatzis D, Lerch A, Hahne F, Stadler MB. 2015. QuasR: quantification and annotation of short reads in R. Bioinformatics 31:1130-1132. doi:10.1093/bioinformatics/btu781

Hendrickson PG, Doráis JA, Grow EJ, Whiddon JL, Lim J-W, Wike CL, Weaver BD, Pflueger C, Emery BR, Wilcox AL, Nix DA, Peterson CM, Tapscott SJ, Carrell DT, Cairns BR. 2017. Conserved roles of mouse DUX and human DUX4 in activating cleavage-stage genes and MERVL/HERVL retrotransposons. Nat Genet 49:925-934. doi:10.1038/ng.3844

Hubner NC, Bird AW, Cox J, Splettstoesser B, Bandilla P, Poser I, Hyman A, Mann M. 2010. Quantitative proteomics combined with BAC TransgeneOmics reveals in vivo protein interactionsQuantitative BAC interactomics. J Cell Biology 189:739-754. doi:10.1083/jcb.200911091 
Karemaker ID, Vermeulen M. 2018. ZBTB2 reads unmethylated CpG island promoters and regulates embryonic stem cell differentiation. Embo Rep 19. doi:10.15252/embr.201744993

Liu Z, Kraus WL. 2017. Catalytic-Independent Functions of PARP-1 Determine Sox2 Pioneer Activity at Intractable Genomic Loci. Mol Cell 65:589-603.e9. doi:10.1016/j.molcel.2017.01.017

Olivieri D, Papasaikas P, Lukonin I, Rittirsch M, Hess D, Smallwood SA, Stadler MB, Betschinger J. 2020. Repression by hdac3 and dax1 mediates lineage restriction of embryonic stem cells. Biorxiv 2020.09.10.291013. doi:10.1101/2020.09.10.291013

Reynolds N, Latos P, Hynes-Allen A, Loos R, Leaford D, O'Shaughnessy A, Mosaku O, Signolet J, Brennecke $P$, Kalkan T, Costello I, Humphreys P, Mansfield W, Nakagawa K, Strouboulis J, Behrens A, Bertone P, Hendrich B. 2012. NuRD Suppresses Pluripotency Gene Expression to Promote Transcriptional Heterogeneity and Lineage Commitment. Cell Stem Cell 10:583-594. doi:10.1016/j.stem.2012.02.020

Ritchie ME, Phipson B, Wu D, Hu Y, Law CW, Shi W, Smyth GK. 2015. limma powers differential expression analyses for RNA-sequencing and microarray studies. Nucleic Acids Res 43:e47e47. doi:10.1093/nar/gkv007

Robinson MD, Oshlack A. 2010. A scaling normalization method for differential expression analysis of RNA-seq data. Genome Biol 11:R25. doi:10.1186/gb-2010-11-3-r25

Schnütgen F, Hansen J, De-Zolt S, Horn C, Lutz M, Floss T, Wurst W, Noppinger PR, Melchner $\mathrm{H}$ von. 2008. Enhanced gene trapping in mouse embryonic stem cells. Nucleic Acids Res 36:e133-e133. doi:10.1093/nar/gkn603

Tyanova S, Temu T, Cox J. 2016. The MaxQuant computational platform for mass spectrometry-based shotgun proteomics. Nat Protoc 11:2301-2319. doi:10.1038/nprot.2016.136

Villegas $F$, Lehalle $D$, Mayer $D$, Rittirsch $M$, Stadler $M B$, Zinner $M$, Olivieri $D$, Vabres $P$, Duplomb-Jego L, Bont ESJMD, Duffourd Y, Duijkers F, Avila M, Geneviève $D$, Houcinat N, Jouan T, Kuentz P, Lichtenbelt KD, Thauvin-Robinet C, St-Onge J, Thevenon J, Gassen KLI van, Haelst $M$ van, Koningsbruggen $S$ van, Hess D, Smallwood SA, Rivière J-B, Faivre L, Betschinger J. 2019. Lysosomal Signaling Licenses Embryonic Stem Cell Differentiation via Inactivation of Tfe3. Cell Stem Cell 24:257-270.e8. doi:10.1016/j.stem.2018.11.021

Zhang Y, Liu T, Meyer CA, Eeckhoute J, Johnson DS, Bernstein BE, Nusbaum C, Myers RM, Brown M, Li W, Liu XS. 2008. Model-based Analysis of ChIP-Seq (MACS). Genome Biol 9:R137. doi:10.1186/gb-2008-9-9-r137 
Figure 1
A

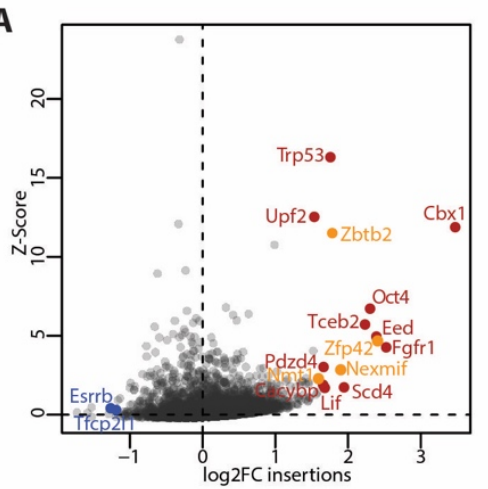

$\mathbf{E}$

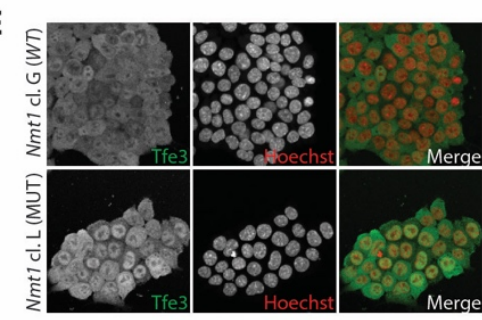

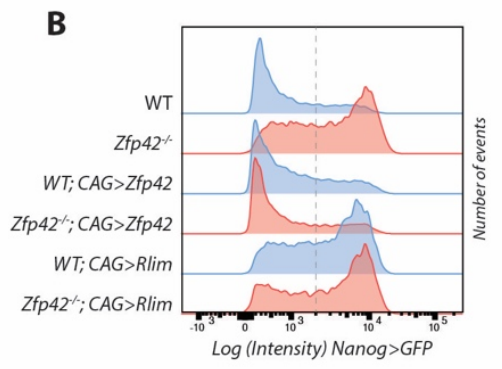
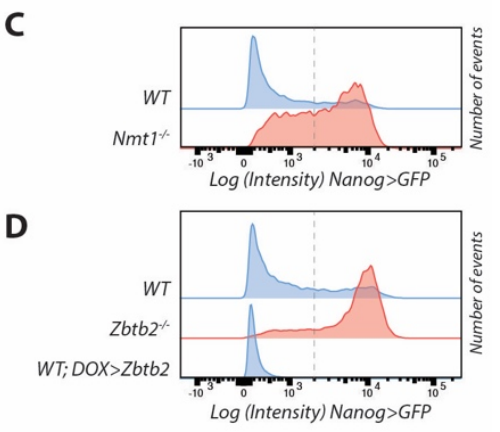

F

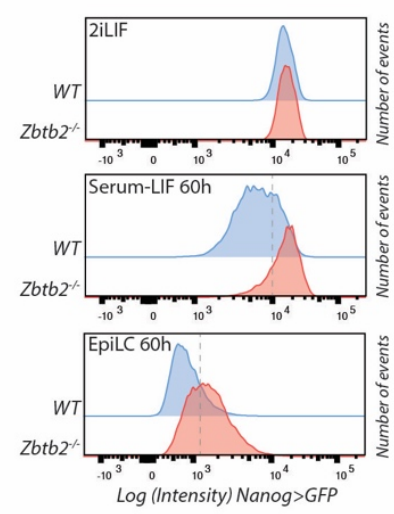

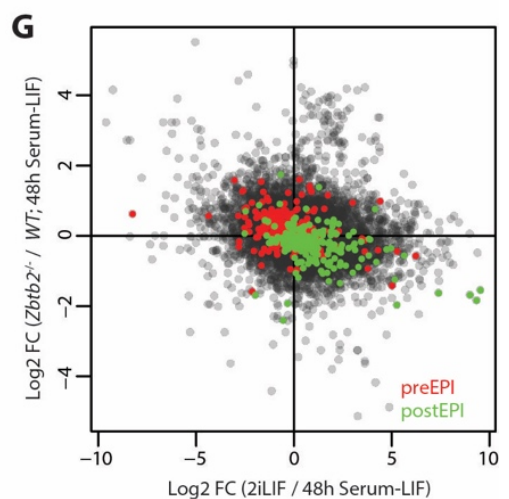

4 Figure 1: Screen results and validation.

5 (A) Z-scores and enrichment fold-changes of retroviral insertions within the gene bodies of 6 indicated genes

7 (B-D,F) Nanog >GFP intensities after 3 days in N2B27 +CHIR +bFGF (B-D) and as indicated (F) 8 of indicated genotypes and treatments. Dashed lines indicate the thresholds for 9 quantifications presented in Fig. S1D,F.

10 (E) Tfe3 immunofluorescence in $\mathrm{Nmt1}^{-/}$and WT mESCs in 2iLIF. Nuclei were counterstained 11 with Hoechst.

12 (G) Scatterplot of log2 fold-changes (FC) in gene expression of indicated contrasts. Green 13 labels post-implantation and red pre-implantation epiblast specific genes (Boroviak et al., 14 2015). 
Figure S1

A

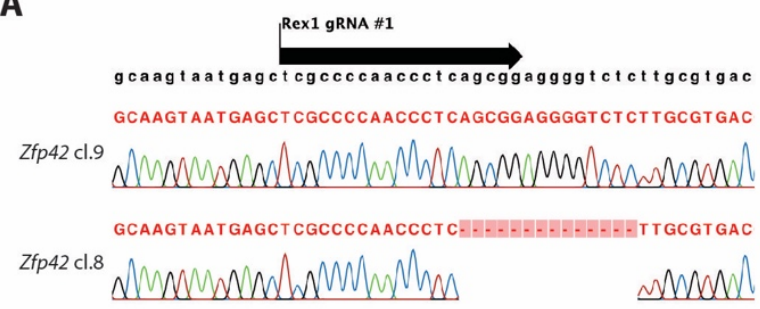

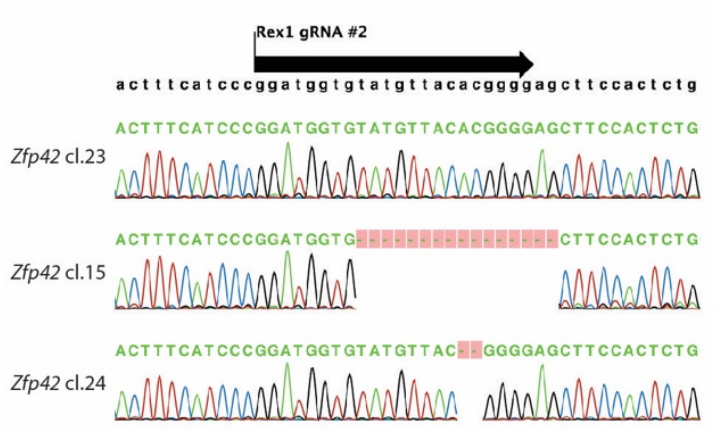

B Nmt1 gRNA 2+6 C Zbtb2 gRNA 4+6 Zbtb2 gRNA $5+8$

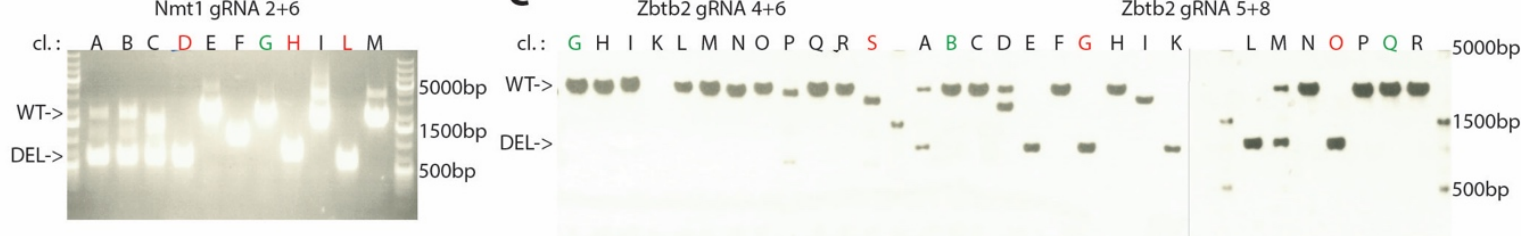

G
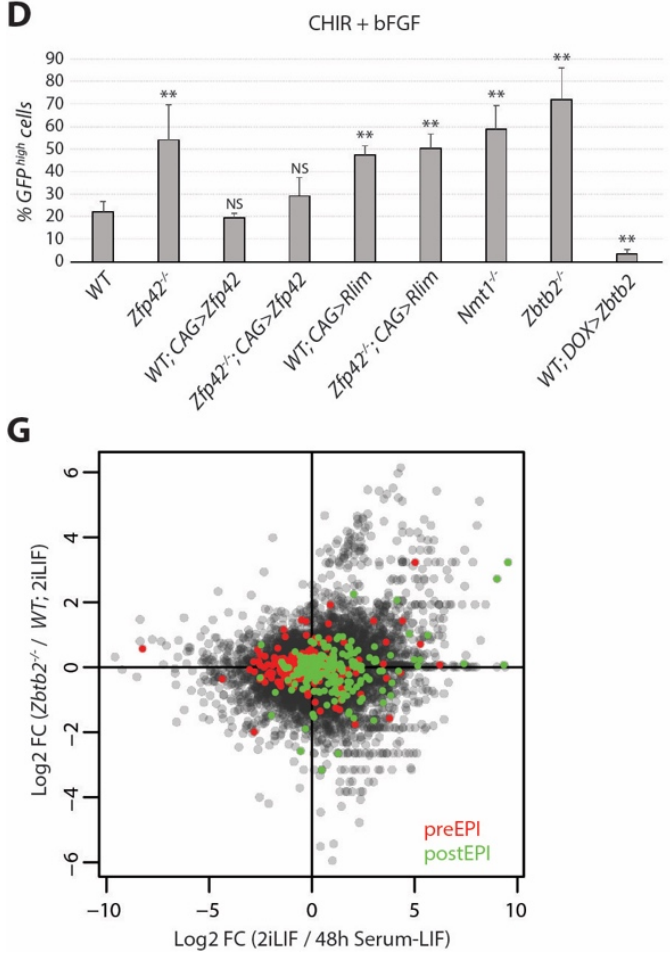

E
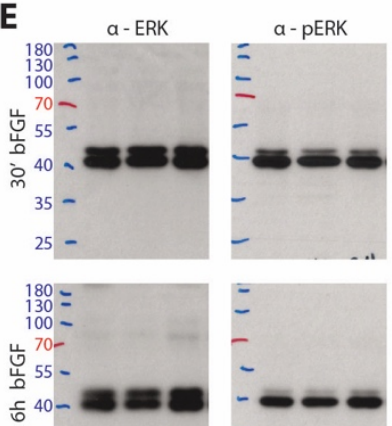

$\mathbf{F}$
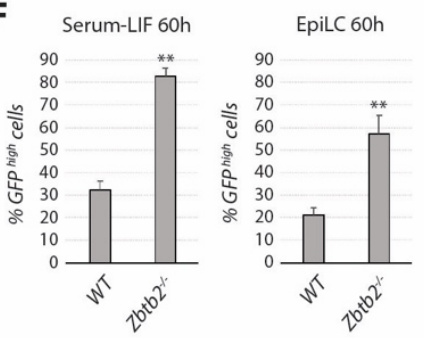

Supplementary Figure S1: Related to Figure 1.

3 (A) Chromatograms of Zfp42/- and WT sibling clones.

$4 \quad(B, C)$ Genotyping PCR of Nmt1 and Zbtb2 knockout and sibling control clones used in this 5 study.

6 (D,F) Average and standard deviation (SD) of Nanog $>$ GFP-high cells of biological triplicates 7 quantified as in Fig.1B, C, D (D). and Fig.1F (F). ${ }^{* *}$ indicates $p$-values $<0.001$ and NS p8 values $>0.1$ compared to corresponding $W T$ controls.

9 (E) Anti-ERK and anti-phospho-ERK western-blot of lysates from $\mathrm{Nmt1}^{-1-}$ and $W T$ clones.

10 (G) Scatterplot of log2FCs in gene expression of indicated contrasts. Green labels post11 implantation and red pre-implantation epiblast specific genes (Boroviak et al., 2015). 
Figure 2
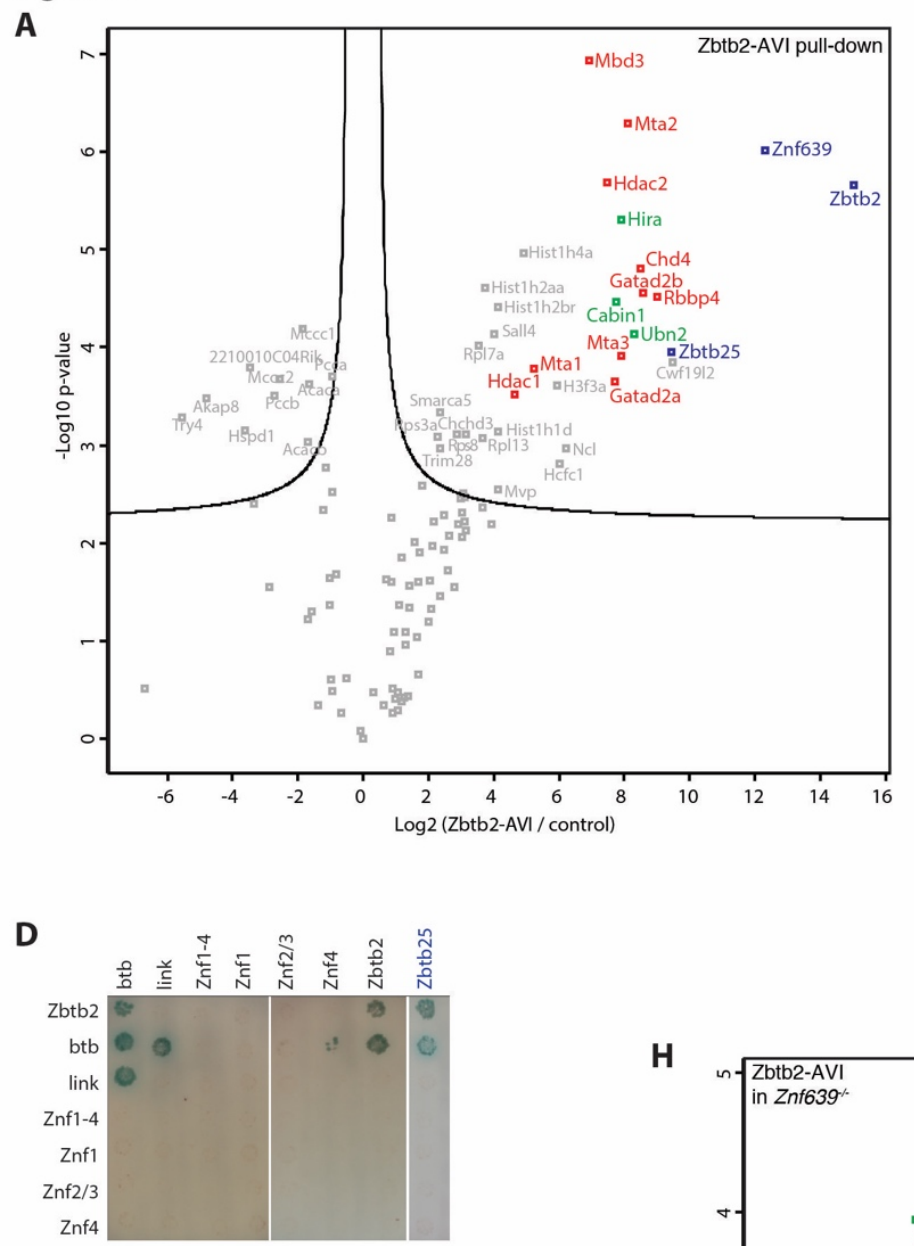

E

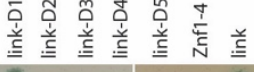
btb btb-link
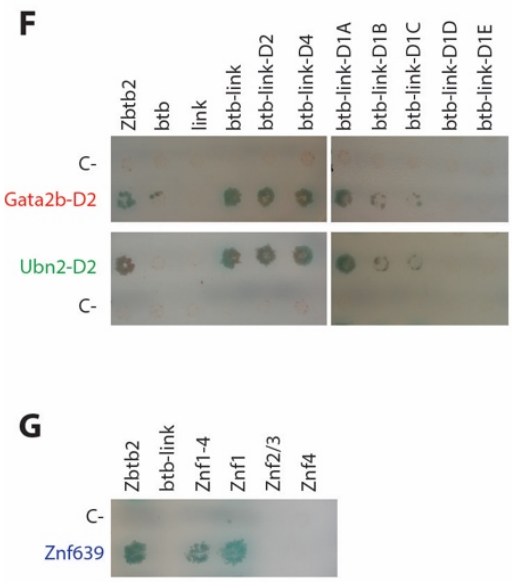

I
B

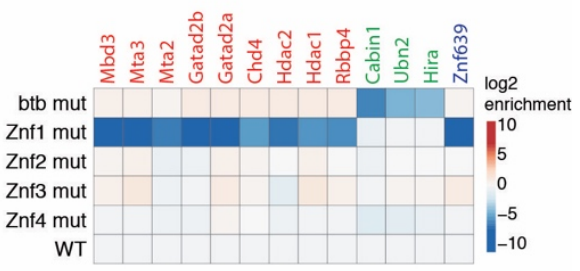

C

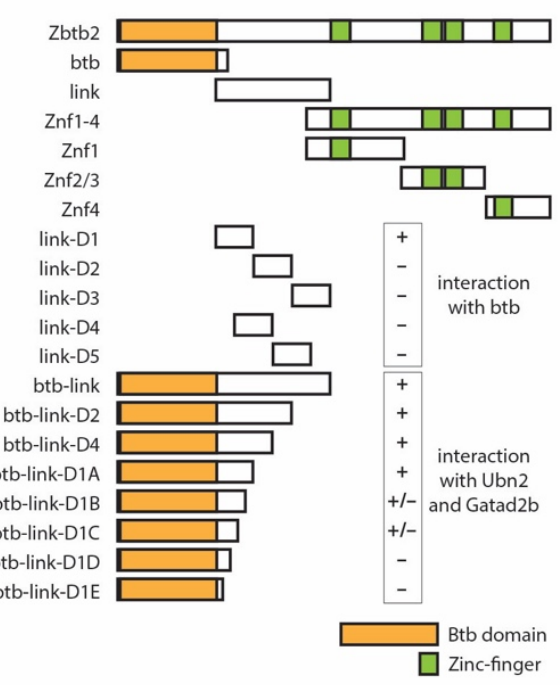

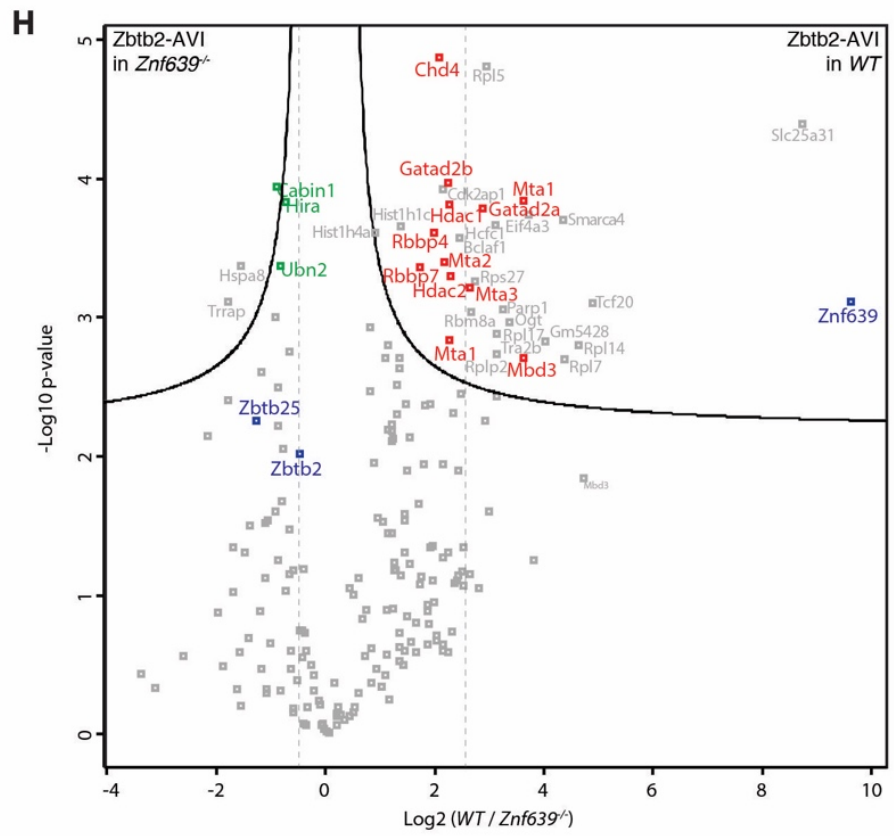

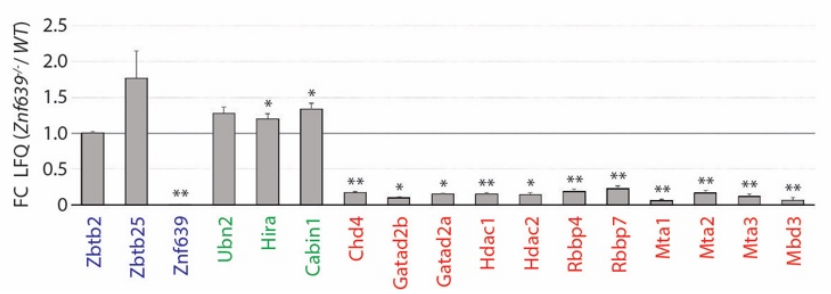


1 Figure 2: An extended btb domain binds UBN2 and GATAD2B; NuRD interaction is stabilized 2 by ZNF639.

3 (A) Volcano plot of protein enrichments in AP-MS of ZBTB2-AVI compared to control BirA4 expressing cells in 2iLIF. ZBTB2 and partner TFs are indicated in blue, NuRD subunits in red, 5 and HiRA subunits in green.

6 (B) Bait-normalized log2 fold enrichment of ZNF639, HiRA subunits and NuRD subunits in AP7 MS of ZBTB2-AVI alleles with indicated mutation compared to wildtype ZBTB2-AVI in Zbtb2-/$8 \quad$ Zbtb25\% cells in 2iLIF.

9 (C) Diagram of ZBTB2 constructs used for $\mathrm{Y} 2 \mathrm{H}$ analysis; + and - indicates positive and negative 10 interactions as in (E,F).

11 (D-G) Colony growth of strains expressing indicated full length and deletion proteins. Bait constructs are vertical $(\mathbf{D}, \mathbf{E})$ and horizontal $(\mathbf{F}, \mathbf{G})$, and prey constructs are horizontal $(\mathbf{D}, \mathbf{E})$ and 13 vertical $(\mathbf{F}, \mathbf{G})$.

$14(\mathbf{H}, \mathbf{I})$ Volcano plot of protein enrichments in AP-MS of ZBTB2-AVI in WT compared to Znf63915 /- cells in 2iLIF. Color code as in Fig. 2A (H). Bait-normalized interaction changes in WT 16 compared to Znf639 mutant cells (I). * indicates $p$-value $<0.01$ and ** p-value $<0.001$. 
Figure S2

A

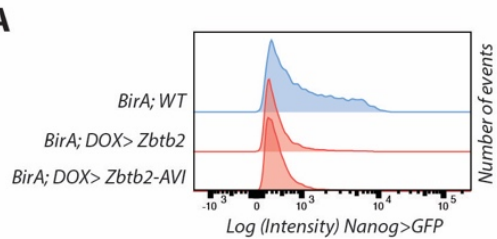

D

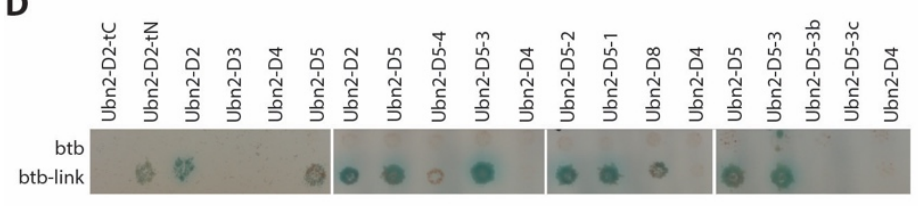

E

Ubn2-D Ubn2-D2 Ubn2-D2-tC Ubn2-D2-tN

Ubn2-D4

Ubn2-D5

Ubn2-D5-1

Ubn2-D5-2

Ubn2-D5-3

Ubn2-D5-4

Ubn2-D

Ubn2-D5-3b

Ubn2-D5-3c

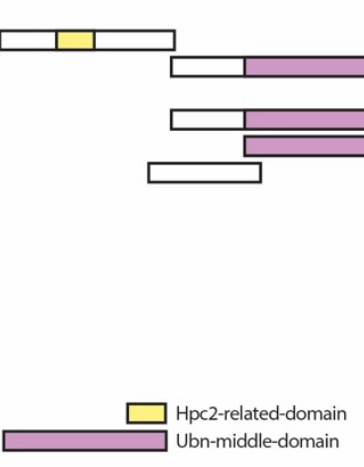

H

Gatad2b
Gatad2b-D1 Gatad2b-D2 Gatad2b-Znf Gatad2b-Cter

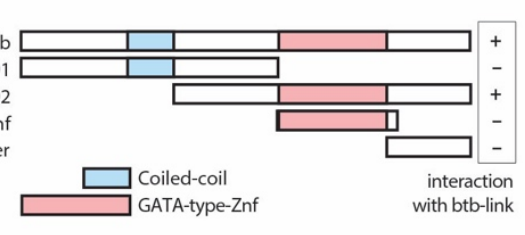

B

Zbtb25 gRNA $2+4$ cl. : wt wt wt 213335 wt

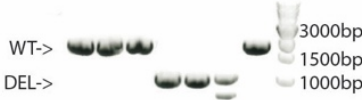

-500 bp
C

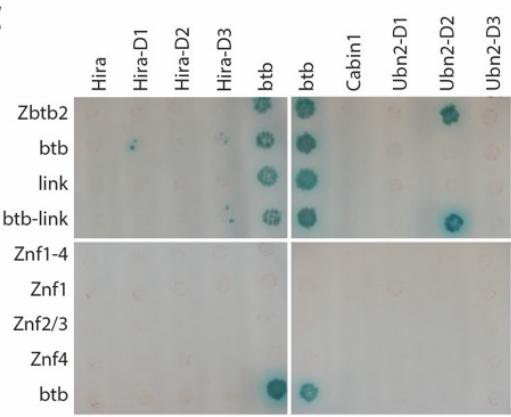

$\mathbf{F}$

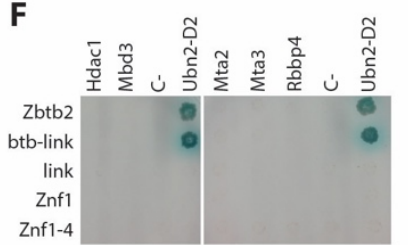

G
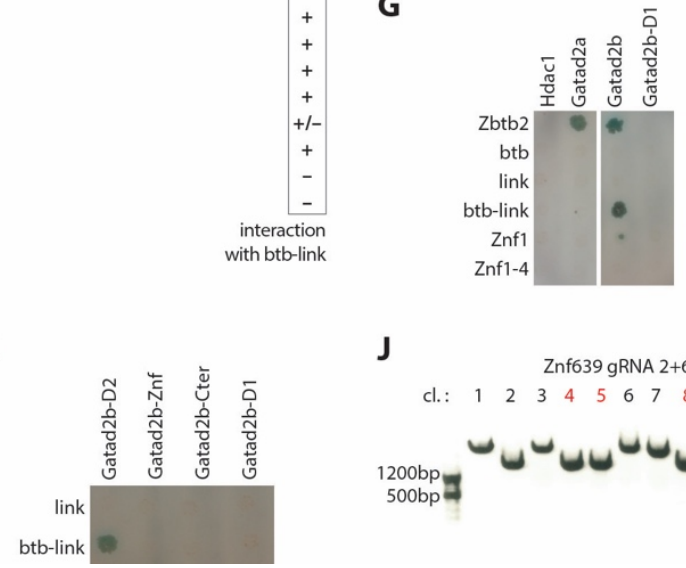

J

Znf639 gRNA 2+6 cl.: $\quad \begin{array}{llllllllll}1 & 2 & 3 & 4 & 5 & 6 & 7 & 8 & 9 & 10\end{array}$

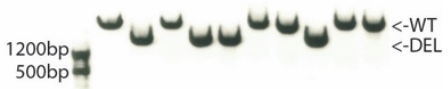

1

\section{Supplementary Figure S2: Identification of the ZBTB2-interacting subunits and domains.} Related to Figure 2.

(A) Nanog $>$ GFP intensities upon ZBTB2 or ZBTB2-AVI induction for 3 days in N2B27 +CHIR $+\mathrm{bFGF}$.

(B) Zbtb25 genotyping PCR of Zbtb2\%; Zbtb25\% clones; red labels clones used in this study. $(C, D, F, G, I)$ As in Fig. 2D (C,F,G) and Fig. $2 E(D, I)$.

$(\mathrm{E}, \mathrm{H})$ Diagram of Ubn2 (E) and Gatad2b $(\mathrm{H})$ constructs used for $\mathrm{Y} 2 \mathrm{H}$ analysis.

(J) Genotyping PCR of Znf639 mutant clones; red labels clones used in this study. 
Figure 3

A

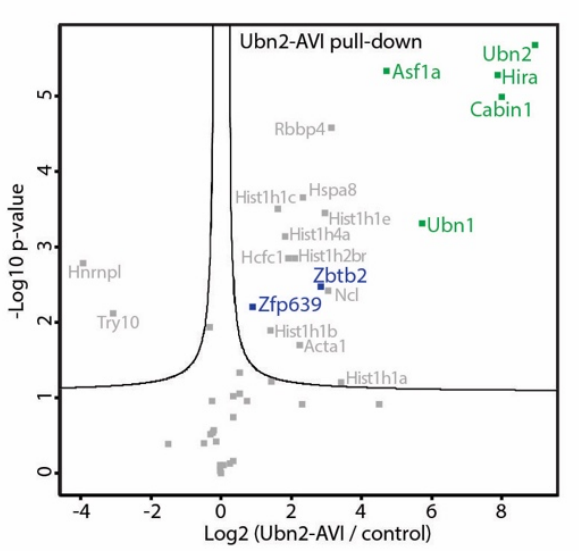

B

D

1

2

3

4

5

6

7

8

9

10

11

12

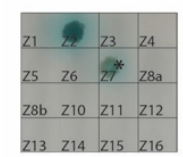

C

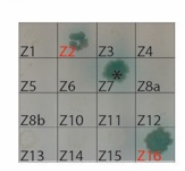

Ubn2-D2
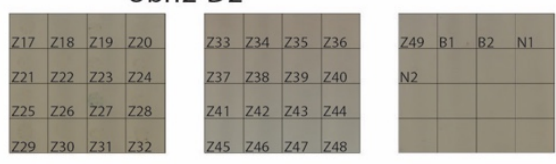

Gatad2b-D2
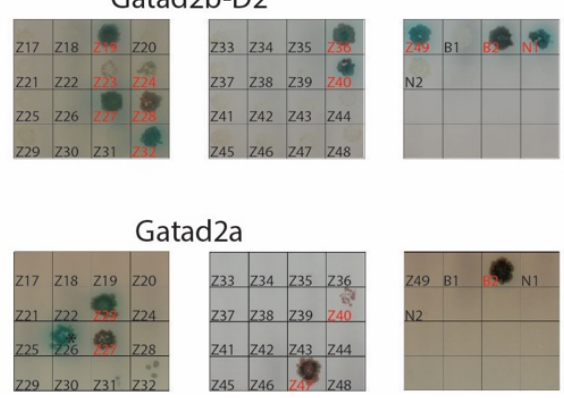
green.
E

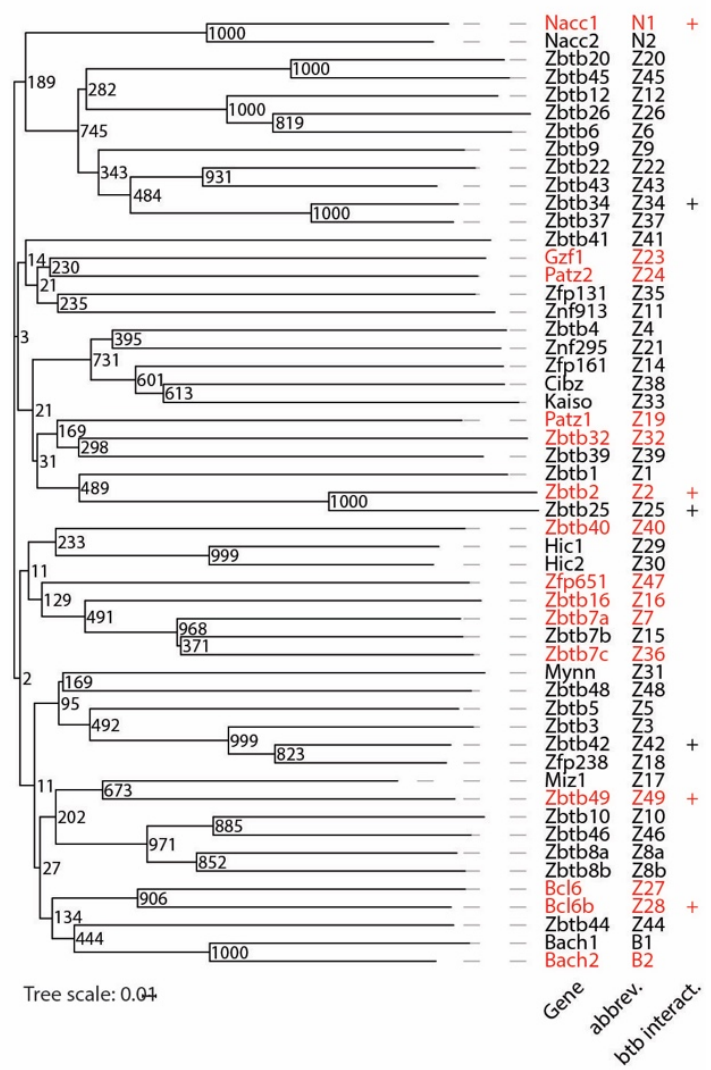

F btb

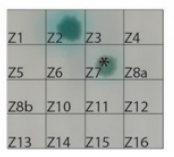

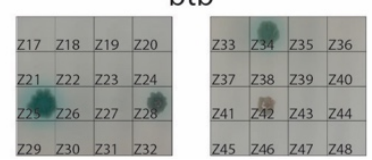

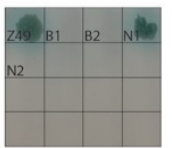

Figure 3: Recruitment of HiRA is a unique property of ZBTB2, while GATAD2A/B interaction is a conserved feature of TF-associated btb domains.

(A) Volcano plot of protein enrichments in AP-MS of UBN2-AVI compared to control BirAexpressing cells in 2iLIF. ZBTB2 and partner TFs are indicated in blue and HiRA subunits in

(B-D,F) Colony growth of strains expressing extended btb domains of Zbtb (Z\#), Bach (B\#) and Nacc (N\#) bait constructs, and Ubn2-D2 (B), Gatad2b-D2 (C), Gatad2a (D) and ZBTB2 btb domain (F) prey constructs. GATAD2A/B interactors are indicated in red. Asterisks mark autoactivating bait constructs. (E) Phylogenetic tree of TF-associated btb domains with bootstrap values. GATAD2A/B interactors, based on Fig. 2C,D and (Masuda et al., 2016) are indicated in red. + indicates btb domains dimerizing with ZBTB2. 
Figure S3

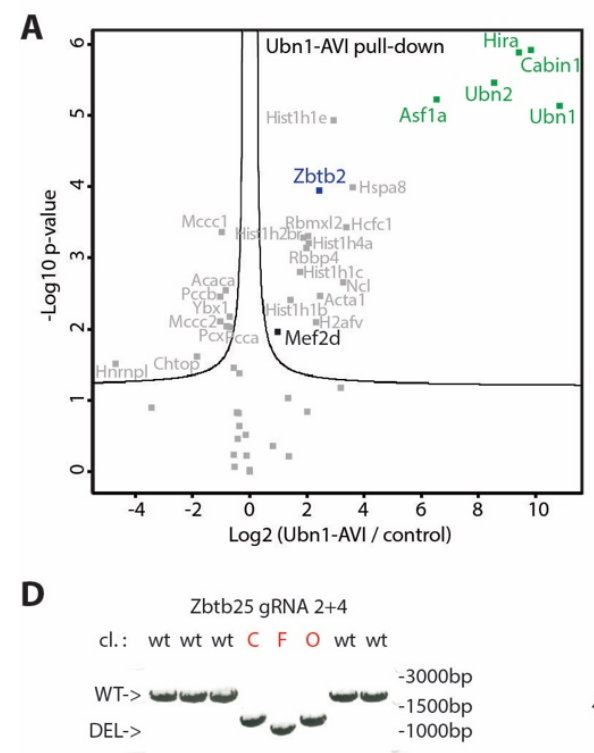

B
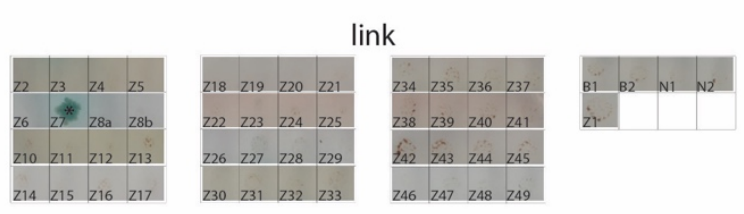

C
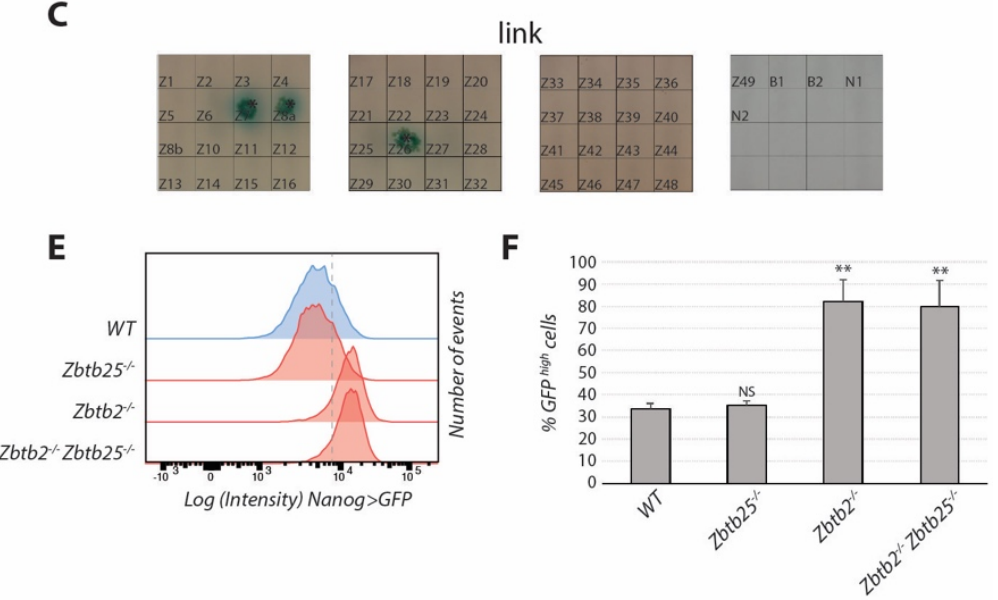

2 Supplementary Figure S3: Lack of genetic interaction between ZBTB2 and ZBTB25. Related 3 to Figure 3.

4 (A) Volcano plot of protein enrichments in AP-MS of UBN1-AVI compared to control BirA5 expressing cells in 2iLIF. ZBTB2 is indicated in blue and HiRA subunits in green.

$6 \quad(B, C)$ Colony growth of control matings for experiments presented in Fig.3B,C,F (B) and in 7 Fig.3D (C) using ZBTB2's link region as control bait construct.

8 (D) Zbtb25 genotyping PCR of $Z b t b 25^{\%}$ clones; red labels clones used in this study.

9 (E,F) Nanog $>$ GFP intensities of indicated genotypes after 60h in Serum-LIF (E). Dashed line 10 indicates the threshold for quantification of GFP-high cells as average and SD of biological 11 triplicates $(\mathbf{F}){ }^{* *}$ indicates $p$-values $<0.001$ and NS $p$-values $>0.1$ compared to the $W T$ control. 
Figure 4

A

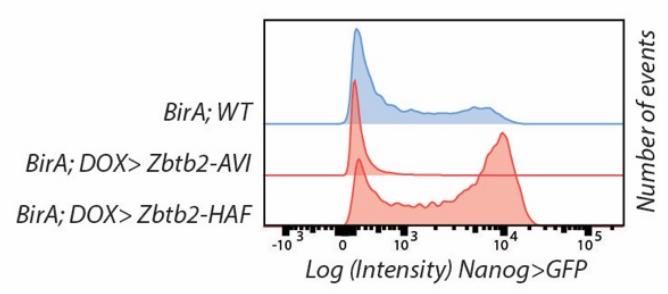

B

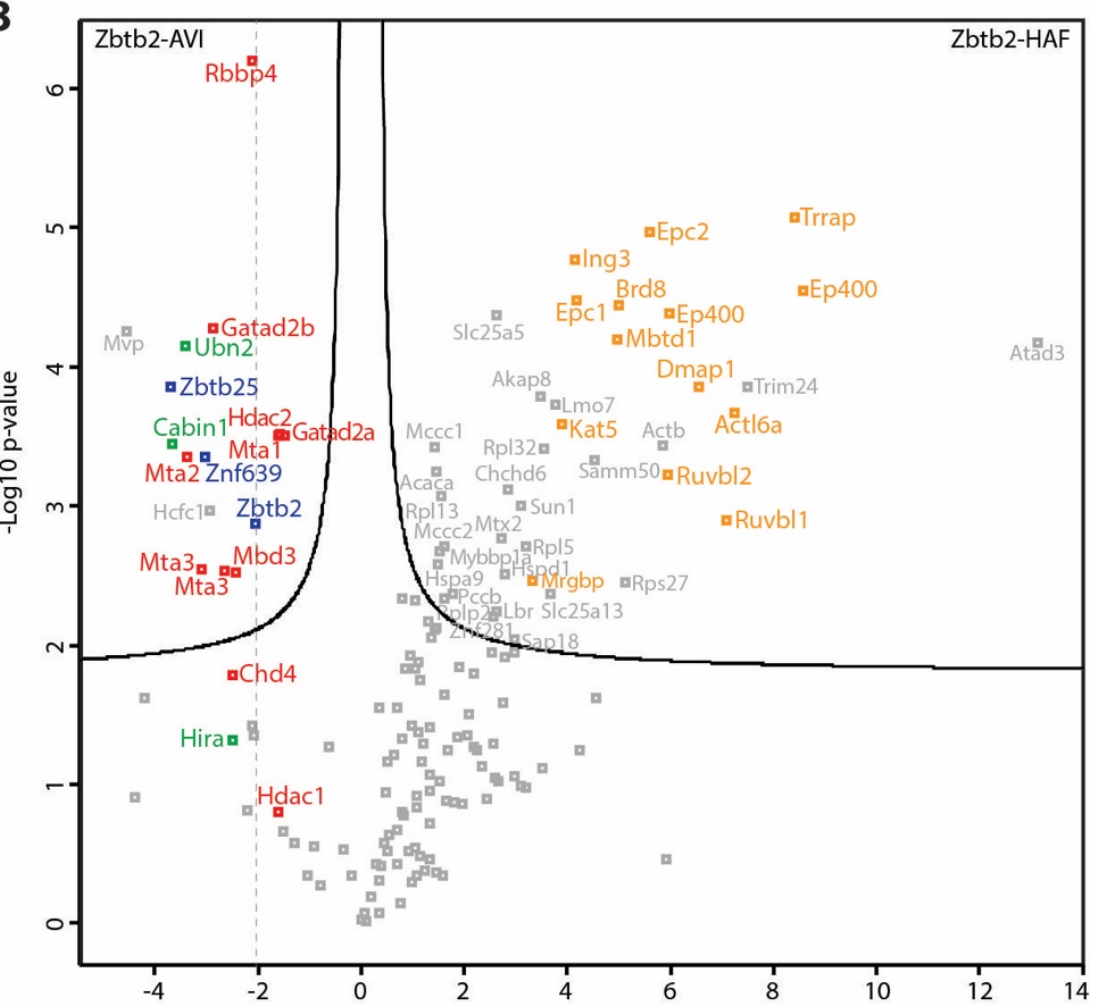

Log2 (Zbtb2-HAF / Zbtb2-AVI)

2 Figure 4: A dominant negative Zbtb2 construct stabilizes the interaction with the Ep400 3 complex.

4 (A) Nanog>GFP intensities upon Zbtb2-AVI or Zbtb2-HAF induction for 3 days in N2B27 +CHIR 5 +bFGF.

6 (B) Volcano plot of protein enrichments in AP-MS of ZBTB2-HAF compared to ZBTB2-AVI.

7 ZBTB2 and partner TFs are indicated in blue, NuRD subunits in red, HiRA subunits in green, 8 and Ep400 subunits in orange. 


\section{Figure S4}

A

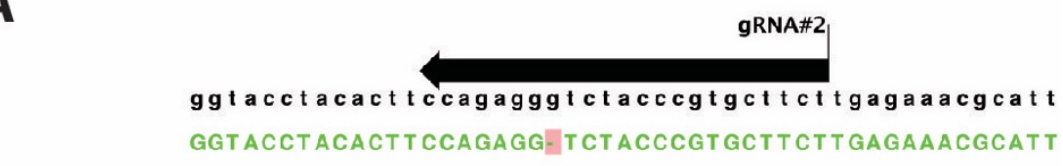

Ubn2 cl.10
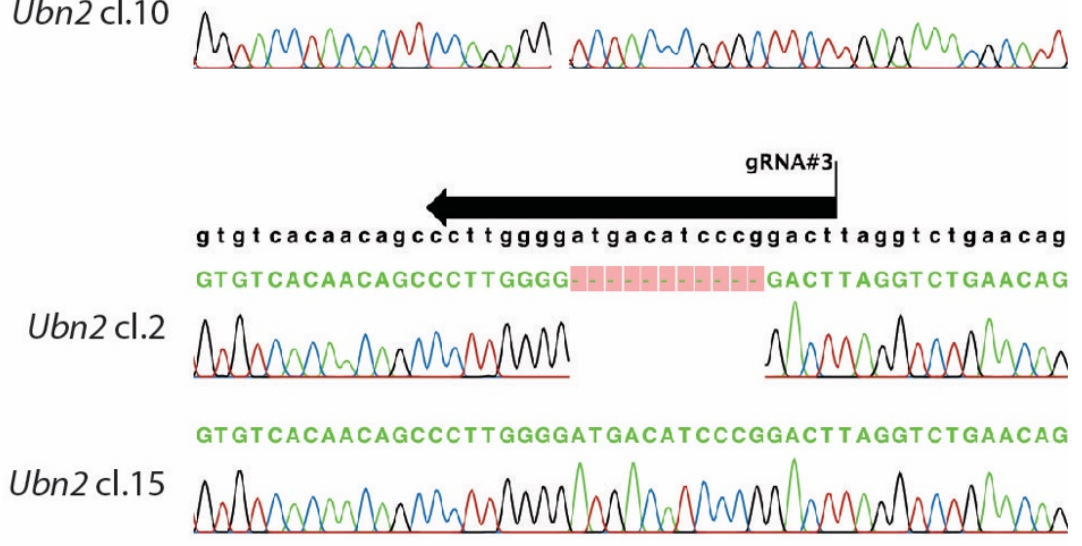

B

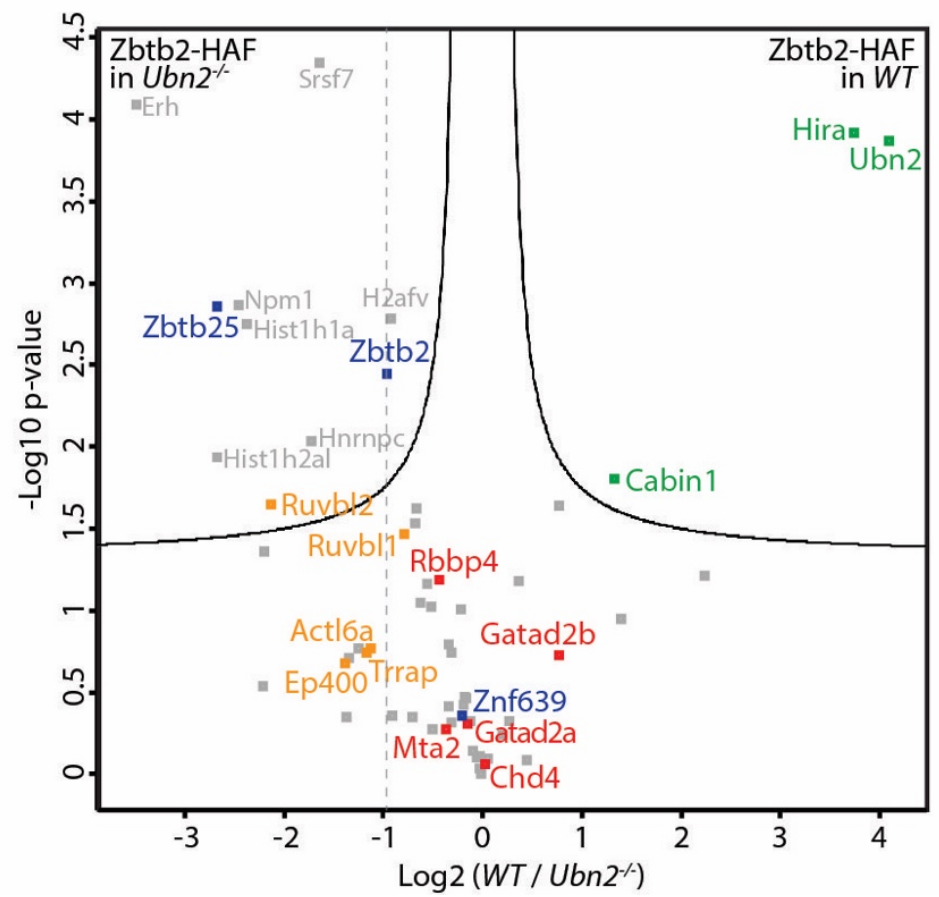

Supplementary Figure S4: The Ep400 interaction is not mediated by the HiRA complex.

\section{Related to Figure 4.}

4 (A) Chromatograms of $U b n 2^{-/-}$and sibling WT clones.

5 (B) Volcano plot of protein enrichments in AP-MS of ZBTB2-HAF in WT compared to Ubn2 $2^{-1}$ 6 cells. ZBTB2 and partner TFs are indicated in blue. The dashed line marks enrichment of the 7 ZBTB2-HAF bait protein which is similar to that of NuRD subunits in red and Ep400 subunits 8 in orange, while interaction with HiRA subunits in green is comparatively reduced in Ubn2 9 mutant cells. 
Figure 5

A

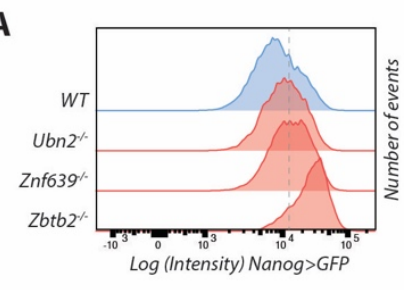

B

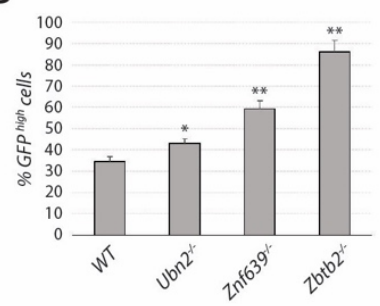

C

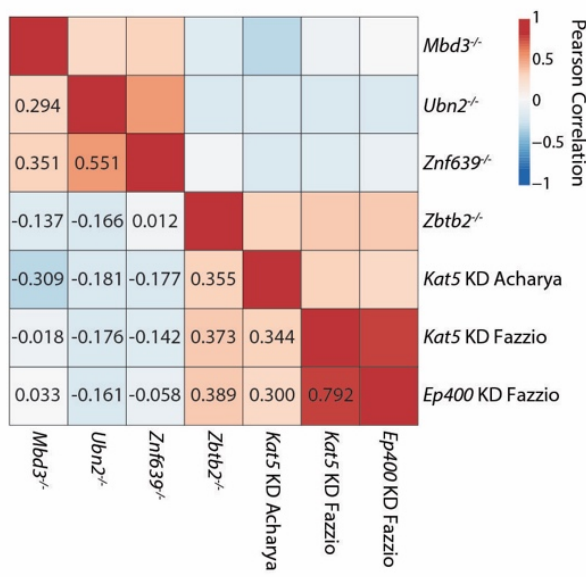

$\mathbf{F}$

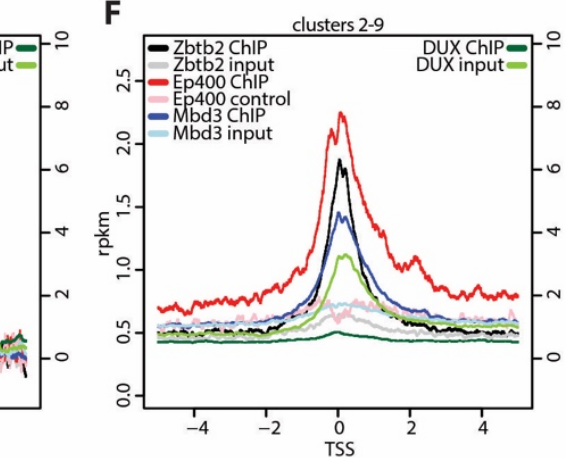

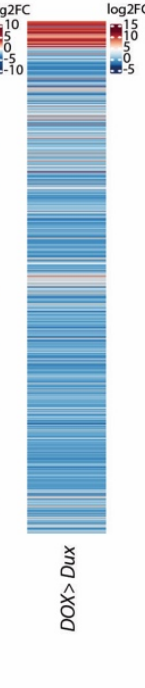

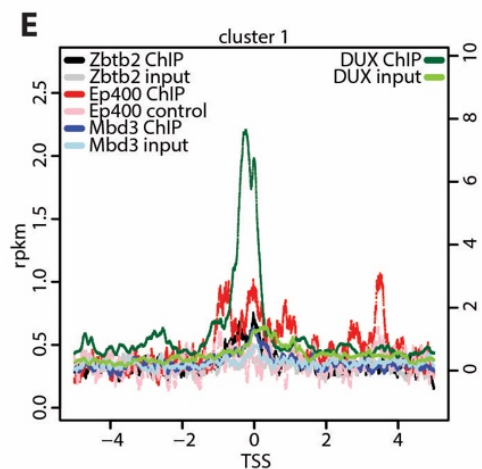

Figure 5: Ep400 and ZNF639/NuRD/HiRA constitute independent functional modules.

(A,B) Nanog $>$ GFP intensities 3 days in Serum-LIF of indicated genotypes (A). Dashed line indicates the threshold for quantification of GFP-high cells as average and SD of biological triplicates (B). * indciates $p$-values $<0.01$ and ${ }^{* *} p$-values $<0.001$ compared to the $W T$ control. (C) Pairwise Pearson correlations of differential gene expression relative to respective control cells after $48 \mathrm{~h}$ in Serum-LIF for Ubn2 ${ }^{--}$, Znf639 ${ }^{-/}$and $\mathrm{Zbtb2}^{-/}$mutant cells, and for $\mathrm{Mbd3}^{-/-}$ (Reynolds et al., 2012), Kat5 KD (Acharya et al., 2017; Fazzio et al., 2008) and Ep400 KD (Fazzio et al., 2008) in Serum-LIF. Only genes deregulated in $\mathrm{Zbtb}^{-/-}$cells were considered (1420 genes).

(D) k-means clustering of differential gene expression as in Fig. $2 \mathrm{C}$ and upon Dux overexpression (Hendrickson et al., 2017).

13 (E,F) ZBTB2 (Karemaker and Vermeulen, 2018), EP400 (Chen et al., 2015) and MBD3

14 (Bornelöv et al., 2018) (left scale), and DUX (Hendrickson et al., 2017) (right scale) ChIP-seq reads per kilobase of transcript per million mapped reads $(\mathrm{rpkm})$ of centered on transcriptional start sites (TSSs) of cluster 1 (E) and cluster 2-9 genes (F). 
Figure S5

A

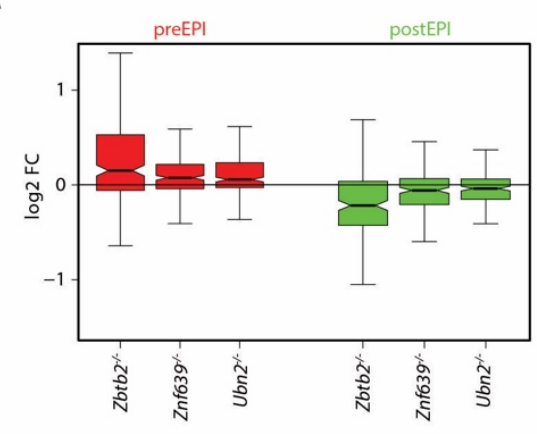

B

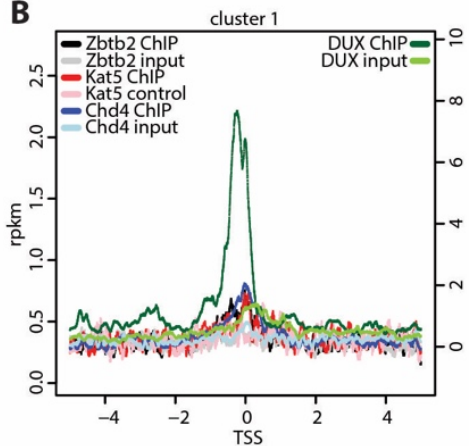

C

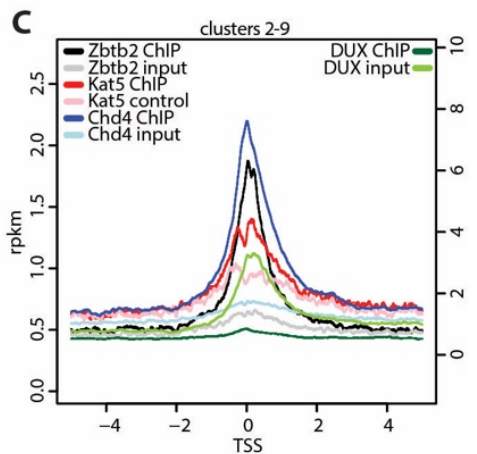

D
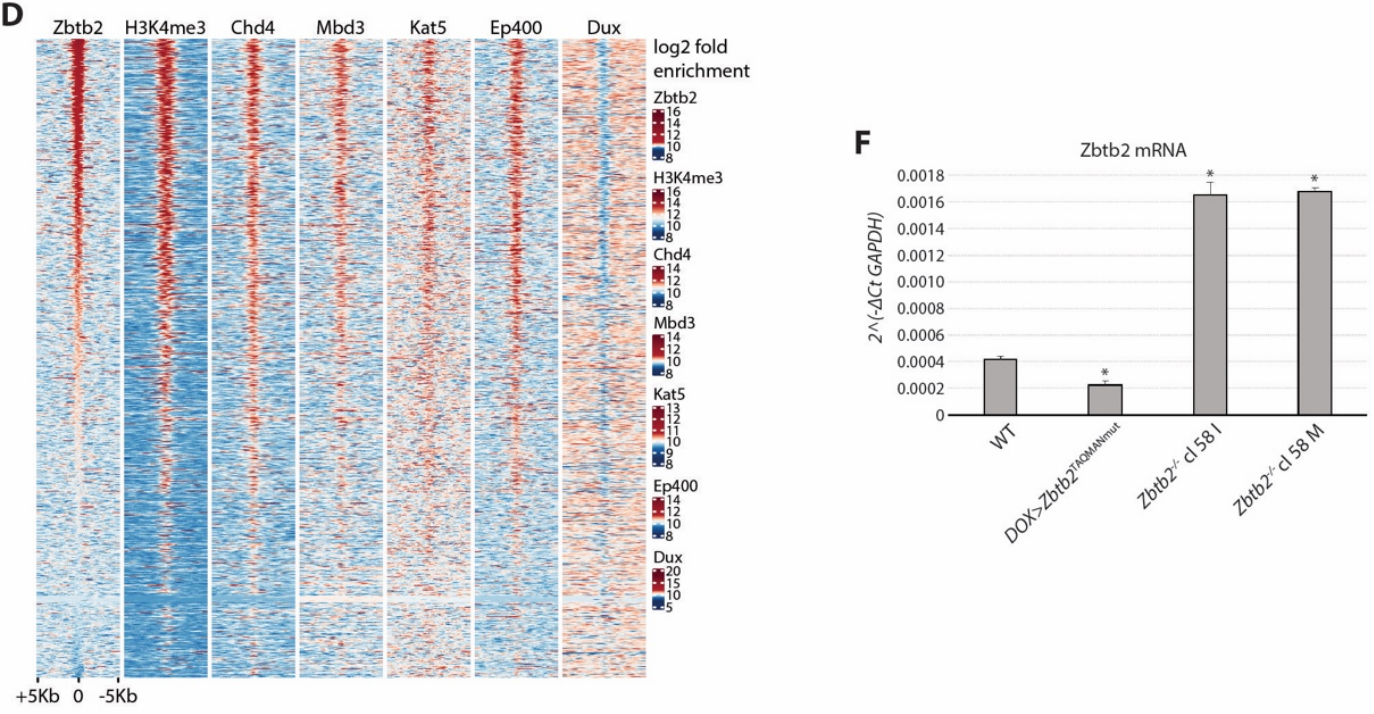

E

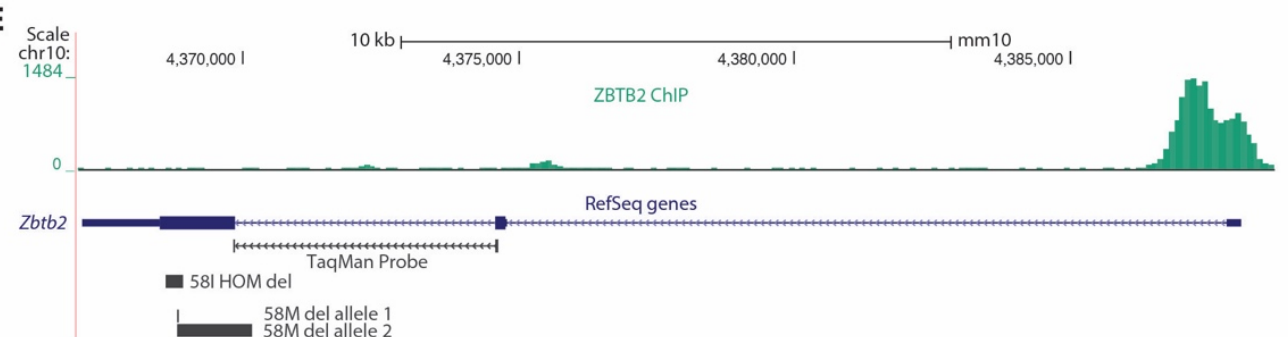

Supplementary Figure S5: ZBTB2 binds and represses its own promoter. Related to Figure 5.

(A) Boxplot of $\log 2$ fold differential expression after $48 \mathrm{~h}$ in Serum-LIF of post-implantation and pre-implantation epiblast specific genes (Boroviak et al., 2015) in indicated genotypes compared to $W T$ cells.

7 (B,C) ZBTB2 (Karemaker and Vermeulen, 2018), KAT5 (Chen et al., 2015) and CHD4 (Bornelöv 8 et al., 2018) (left scale), and DUX (Hendrickson et al., 2017) (right scale) ChIP-seq rpkm centered on TSSs of cluster 1 (B) and cluster 2-9 genes (C).

10 (D) Heatmap of ZBTB2 (Karemaker and Vermeulen, 2018), H3K4me3 (Liu and Kraus, 2017), CHD4, MBD3 (Bornelöv et al., 2018), KAT5, EP400 (Chen et al., 2015) and DUX (Hendrickson et al., 2017) log2 fold ChIP-seq enrichment over respective controls at accessible (ATACseq, not shown) TSSs. 
1 (E) Diagram of the Zbtb2 locus, showing the ZBTB2 peak at the TSS, and the deletions in the $2 \mathrm{Zbtb2^{-/ }}$ clones $58 \mathrm{I}$ and $58 \mathrm{M}$ that give rise to transcripts that are detectable by qPCR probes 3 against endogenous Zbtb2.

4 (F) Average and SD of technical triplicates of endogenous Zbtb2 relative to GAPDH 5 transcription in WT cells, WT cells over-expressing a Zbtb2 construct that cannot be detected 6 by the qPCR probe (TAQMANmut) and the $\mathrm{Zbtb}^{-/}$clones depicted in Fig. S2E. * indicates p7 values $<0.01$ relative compared to $W T$.

8

9 SUPPPLEMENTARY TABLES

10

11 Supplementary Table S1: Screen results

12 Supplementary Table S2: CRISPR KO and genotyping strategies; cell lines

13 Supplementary Table S3: Gene expression tables with clusters and post- and pre14 implantation genes

15 Supplementary Table S4: AP-MS bait sequences and Y2H constructs

16 Supplementary Table S5: AP-MS results tables 